\title{
From the Fan-KKM principle to extended real-valued equilibria and to variational-hemivariational inequalities with application to nonmonotone contact problems
}

\author{
Joachim Gwinner ${ }^{1 *}$ \\ Dedicated to the memory of Professor Werner Oettli
}

*Correspondence:

joachim.gwinner@unibw-muenchen.de ${ }^{1}$ Fakultät für Luft- und Raumfahrttechnik, Universität der Bundeswehr München, Neubiberg/München, Germany

\section{(2) Springer}

\begin{abstract}
This paper starts off by the celebrated Knaster-Kuratowski-Mazurkiewicz principle in the formulation by Ky Fan. We provide a novel variant of this principle and build an existence theory for extended real-valued equilibrium problems with general, then monotone and pseudomonotone bifunctions. We develop our existence theory first in general topological vector spaces, then in reflexive Banach spaces, where we investigate the issue of coerciveness for existence on unbounded sets. Thereafter we use the Clarke generalized differential calculus for locally Lipschitz functions and derive existence results for nonlinear variational-hemivariational inequalities and hemivariational quasivariational inequalities. As application, we treat a unilateral contact problem in solid mechanics with nonmonotone friction.
\end{abstract}

MSC: 47J20; 47H05; 49J40; 49J52; 74G22; 74M10; 74M15

Keywords: Extended real-valued equilibria; Pseudomonotone bifunction; Hemivariational inequality; Unilateral contact problem; Non-monotone friction

\section{Introduction}

This paper starts off by the celebrated Knaster-Kuratowski-Mazurkiewicz (KKM) principle in the formulation by Ky Fan [22, 23], which is known to be equivalent to topological fixed point theorems [28]. First, we provide a new refined version of the Fan-KKM principle, which relies on a general coercivity condition and unifies some results on this topic in $[17,21]$. Based on this refined version of the Fan-KKM principle, we build a broad existence theory for extended real-valued equilibrium problems with general bifunctions, then with bifunctions of monotone type, including monotone and (Brèzis or topologically) pseudomonotone bifunctions. We show how by this equilibrium theory existence results for variational inequalities of monotone type with set-valued operators can be

(c) The Author(s) 2022. This article is licensed under a Creative Commons Attribution 4.0 International License, which permits use, sharing, adaptation, distribution and reproduction in any medium or format, as long as you give appropriate credit to the original author(s) and the source, provide a link to the Creative Commons licence, and indicate if changes were made. The images or other third party material in this article are included in the article's Creative Commons licence, unless indicated otherwise in a credit line to the material. If material is not included in the article's Creative Commons licence and your intended use is not permitted by statutory regulation or exceeds the permitted use, you will need to obtain permission directly from the copyright holder. To view a copy of this licence, visit http://creativecommons.org/licenses/by/4.0/. 
derived. The setting of our exposition is first in general topological vector spaces, then in reflexive Banach spaces, where we discuss the issue of coerciveness for existence on unbounded sets in depth. Thereafter we use the Clarke generalized differential calculus [18, Sect. 2.1] for locally Lipschitz functions and obtain existence results for nonlinear variational-hemivariational inequalities and hemivariational quasivariational inequalities. For this latter class of nonlinear variational problems, we present a direct approach to existence results based on equilibrium theory without additional resorting to Banach's contraction theorem as exposed in the monograph of Sofonea and Migorski on variationalhemivariational inequalities [57].

We emphasize that extended real-valued equilibrium problems - a further novel feature of the present paper - cannot be reduced to classical equilibrium problems as pioneered by Blum and Oettli [7]. Although a reduction is trivial for equilibrium problems with convex, lower semicontinuous real-valued functions, the standard trick for extended real-valued convex, lower semicontinuous functions that works with the epigraph of such functions fails, since the transfer to a product space does not preserve compactness and coerciveness properties.

For existence on noncompact sets, we avoid, similarly to [40], coercivity conditions involving interior points, since the topological interior and the algebraic interior of convex sets in infinite-dimensional function spaces may be empty.

As application, we treat a unilateral contact problem in continuum mechanics with nonmonotone friction in the framework of nonlinear variational-hemivariational inequalities. The theory of hemivariational inequalities has been introduced and studied by Panagiotopoulos [50] in the 1980s and 1990s as a generalization of variational inequalities with an aim to model many problems coming from mechanics when the energy functionals are nonconvex; see [25, 44, 51, 56].

The paper is organized as follows. In the next section, we establish our novel variant of the Fan-KKM principle and a general existence theorem for extended real-valued equilibria. In Sect. 3, we are concerned with monotone and pseudomonotone bifunctions, and develop further the existence theory for extended real-valued equilibria. Section 4 provides an application of the equilibrium theory to variational inequalities of monotone type with set-valued operators. Then in Sect. 5, we switch from topological vector spaces to reflexive Banach spaces, and in this setting, we discuss the issue of coerciveness in depth. Next, in Sect. 6, we use the Clarke generalized differential calculus for locally Lipschitz functions and obtain existence results for nonlinear variational-hemivariational inequalities and hemivariational quasivariational inequalities. As a model application, we study a scalar variational problem with the $p$-Laplacian and nonmonotone, possibly set-valued boundary conditions. In Sect. 7, we present an application to a full vectorial contact problem. Here we treat a unilateral contact problems with material nonlinearity and nonmonotone friction. The paper ends in Sect. 8 with some concluding remarks giving an outlook to some related directions of research.

\section{A variant of the Fan-KKM principle and a general existence theorem for extended real-valued equilibria}

Let us give some notations and terminology used in the following. In a topological vector space $E, \mathcal{F}(E)$ is the set of all finite-dimensional subspaces of $E$, ordered by inclusion. Conv $X$ and $\operatorname{cl} X$ or $\bar{X}$ denote the convex hull and the topological closure of a subset $X$ 
of $E$, respectively; $2^{X}$ stands for the power set, the set of all subsets of $X ; \mathcal{N}(X)$ is the set of all (nonempty) finite subsets of $X$, ordered by inclusion. The topological dual of $E$ is written $E^{*}$. A map $M: X \rightarrow 2^{X}$ is called a KKM covering if for every $N \in \mathcal{N}(X)$, we have

$$
\operatorname{Conv} N \subset \bigcup_{x \in N} M(x)
$$

The starting point of our investigations is the following extension of Fan [22, Lemma1] of the classical Knaster-Kuratowski-Mazurkiewicz theorem to an arbitrary Hausdorff topological vector space.

Theorem 2.1 Let $X$ be any nonempty subset of a Hausdorff topological vector space, and let $M: X \rightarrow 2^{X}$ be a KKM covering with closed values. If $M(x)$ is compact for at least one $x \in X$, then $\bigcap\{M(x): x \in X\} \neq \emptyset$.

The proof by Fan uses the finite intersection property of compact sets and employs the Knaster-Kuratowski-Mazurkiewicz theorem; see also [2, Chap. 11] and [5, Appendix B] for this line of proof and the proof of the KKM theorem from Sperner's lemma. Since compactness is a strong assumption in infinite-dimensional spaces, we later relax this assumption to a general coercivity condition, which demands the relative compactness of an appropriate intersection of some sets $M(x)$. Moreover, in view of the later application to existence results for variational inequalities, we have to drop the assumption of closedness of the sets. Instead, similarly as in the approach by Brèzis, Nirenberg, and Stampacchia [9], we work with a refined closedness assumption, which looks for the first look very technical but can be easily verified by topologically pseudomonotone bifunctions, as we will see later.

Here is our novel variant of the Fan-KKM Lemma that encompasses [26, Lemma 1.2] and more recent refinements due to Chowdhury and Tan [17] and Ding and Tarafdar [21].

Theorem 2.2 Let $X$ be a nonempty convex subset of a topological vector space, and let $M: X \rightarrow 2^{X}$ be a KKM covering. Suppose

(i) there exists a convex compact subset $C_{0}$ of $X$ such that $\mathrm{cl}_{X}\left(\bigcap_{x \in C_{0}} M(x)\right)$ is compact,

(ii) for any $N \in \mathcal{N}(X)$ and any $x \in \operatorname{Conv} N$,

$$
M(x) \cap \operatorname{Conv} N \quad \text { is closed in } \operatorname{Conv} N
$$

(iii) for any compact $K \subset X$ and any $N \in \mathcal{N}(X)$, we have, for $D=\operatorname{Conv} N$ and $D=\operatorname{Conv}\left(C_{0} \cup N\right)$,

$$
K \cap D \cap \mathrm{cl}_{X}\left(\bigcap_{x \in D} M(x)\right)=K \cap D \cap \bigcap_{x \in D} M(x)
$$

Then $\bigcap_{x \in X} M(x) \neq \emptyset$.

Proof 1) We apply Theorem 2.1 in a special situation. Fix $N \in \mathcal{N}(X)$ and define the setvalued map $M_{N}$ on Conv $N \subset X$ by $M_{N}(x)=M(x) \cap \operatorname{Conv} N$. Then $M_{N}$ has closed values by (ii) and hence compact values, since $\operatorname{Conv} N$ is compact. Let $\tilde{N} \in \mathcal{N}(\operatorname{Conv} N)$. Then 
$\tilde{N} \in \mathcal{N}(X)$ by convexity of $X$, and Conv $\tilde{N} \subset \bigcup\{M(x): x \in \tilde{N}\}$, since $M$ is a KKM covering by assumption. Moreover, $\operatorname{Conv} \tilde{N} \subset \operatorname{Conv} N$. Therefore $\operatorname{Conv} \tilde{N} \subset \bigcup\left\{M_{N}(x): x \in \tilde{N}\right\}$, and $M_{N}$ is a KKM covering. By Theorem 2.1 there exists $z_{N} \in \operatorname{Conv} N$ such that $z_{N} \in M(x)$ for all $x \in \operatorname{Conv} N$.

2) For any fixed $N \in \mathcal{N}(X)$, let $G=\operatorname{Conv}\left(C_{0} \cup N\right)$. Then $G \subset X$ is compact, since with $N=\left\{x_{1}, \ldots, x_{n}\right\} \subset X$ and the standard simplex $S^{n}$ in $\mathbb{R}^{n+1}, G$ is the continuous image of the compact $C_{0} \times S^{n}$ under the map $\chi$ given by $\chi\left(x_{0}, \lambda_{0}, \lambda_{1}, \ldots, \lambda_{n}\right)=\lambda_{0} x_{0}+\sum_{j=1}^{n} \lambda_{j} x_{j}$ for $x_{0} \in K_{0},\left(\lambda_{0}, \lambda_{1}, \ldots, \lambda_{n}\right) \in S^{n}$.

We want to show that $\bigcap\{M(x): x \in G\} \cap G \neq \emptyset$. To this end, let $y_{H} \in \bigcap\{M(x): x \in$ Conv $H\} \cap \operatorname{Conv} H$, which exists for any $H \in \mathcal{N}(G) \subset \mathcal{N}(X)$ according to the preceding step, and define $Y_{H}=\bigcup\left\{y_{H^{\prime}}: H^{\prime} \supset H, H^{\prime} \in \mathcal{N}(G)\right\}$ for any fixed $H \in \mathcal{N}(G)$. Note that for all $H^{\prime} \supset H$ with $H^{\prime} \in \mathcal{N}(G)$, we have

$$
y_{H^{\prime}} \in \bigcap\left\{M(x): x \in \operatorname{Conv} H^{\prime}\right\} \subset \bigcap\{M(x): x \in \operatorname{Conv} H\} .
$$

Therefore

$$
\text { (*) } \quad Y_{H} \subset \bigcap\{M(x): x \in \operatorname{Conv} H\} \text {. }
$$

Since $Y_{H}$ is contained in the compact set $G \subset X$ and the family $\left\{Y_{H}: H \in \mathcal{N}(G)\right\}$ has the finite intersection property, there exists some $\bar{y} \in G \cap \bigcap\left\{\mathrm{cl}_{X} Y_{H}: H \in \mathcal{N}(G)\right\}$. Now let $y_{1} \in G$ be arbitrary. Consider $H_{1}=\left\{y_{1}, \bar{y}\right\} \in \mathcal{N}(G)$. Then by construction and by $(*)$

$$
\bar{y} \in H_{1} \cap \mathrm{cl}_{X} Y_{H_{1}} \subset \operatorname{Conv} H_{1} \cap \operatorname{cl}_{X}\left[\bigcap\left\{M(x): x \in \operatorname{Conv} H_{1}\right\}\right] .
$$

By (iii) conclude for $K:=G, D:=\operatorname{Conv} H_{1}, \bar{y} \in \bigcap\left\{M(y): y \in \operatorname{Conv} H_{1}\right\} \subset M\left(y_{1}\right)$, and therefore $\bar{y} \in G$ lies in $\bigcap\{M(y): y \in G\}$, as desired.

3) Introduce the family

$$
\mathcal{G}=\left\{G=\operatorname{Conv}\left(C_{0} \cup N\right): N \in \mathcal{N}(X)\right\},
$$

which is ordered by inclusion. According to the preceding step, for any $G \in \mathcal{G}$, there exists some $z_{G} \in \bigcap\{M(x): x \in G\} \cap G$. By construction, $z_{G} \in L \subset X$, where

$$
L:=\operatorname{cl}_{X}\left(\bigcap_{x \in C_{0}} M(x)\right)
$$

is compact by (i).

Define $Z_{G}=\bigcup\left\{z_{G^{\prime}}: G^{\prime}=\operatorname{Conv}\left(C_{0} \cup N^{\prime}\right), N^{\prime} \supset N, N^{\prime} \in \mathcal{N}(X)\right\}$ for any $G=\operatorname{Conv}\left(C_{0} \cup N\right) \in$ $\mathcal{G}$. Then $Z_{G} \subset L$, and the family $\left\{\mathrm{cl}_{X} Z_{G}: G \in \mathcal{G}\right\}$ has the finite intersection property. Hence there exists $\bar{z} \in L$ such that

$$
\bar{z} \in \bigcap_{G \in \mathcal{G}} \operatorname{cl}_{X} Z_{G}
$$


Finally, we show that $\bar{z} \in \bigcap\{M(x): x \in X\}$. Note that for all $N^{\prime} \supset N$ with $N^{\prime} \in \mathcal{N}(X)$ and $G^{\prime}=\operatorname{Conv}\left(C_{0} \cup N^{\prime}\right)$, we have

$$
z_{G^{\prime}} \in \bigcap_{x \in G^{\prime}} M(x) \subset \bigcap_{x \in G} M(x)
$$

Therefore

$$
(* *) \quad Z_{G} \subset \bigcap_{x \in G} M(x) .
$$

Now let $x_{0} \in X$ be arbitrary. Consider $N_{0}=\left\{x_{0}, \bar{z}\right\}$ and $G_{0}=\operatorname{Conv}\left(K_{0} \cup N_{0}\right)$. Then by construction and by $(* *)$

$$
\bar{z} \in L \cap G_{0} \cap \mathrm{cl}_{X} Z_{G_{0}} \subset L \cap G_{0} \cap \operatorname{cl}_{X}\left(\bigcap_{x \in G_{0}} M(x)\right) .
$$

By (iii) conclude with $K:=L, D:=G_{0}, \bar{z} \in \bigcap\left\{M(x): x \in G_{0}\right\} \subset M\left(x_{0}\right)$, and therefore $\bar{z} \in$ $\bigcap\{M(x): x \in X\}$, as desired.

A discussion of our FKKM theorem in relation to the literature is in order. Clearly, the coercivity assumption (i) is implied by the assumption of compactness of $\operatorname{cl}_{X} M\left(x_{0}\right)$ for some $x_{0} \in X$, which is used in [17] and also by Ansari, Lin, and Yao [4] and Kalmoun [36]. Note that in this case, we choose $K=C_{0}=\left\{x_{0}\right\}$ in (i), then $G=\operatorname{Conv}\left(C_{0} \cup N\right)=\operatorname{Conv} \tilde{N}$ with $\tilde{N}=N \cup\left\{x_{0}\right\}$, and so (iii) simplifies to: For any compact $K \subset X$ and any $N \in \mathcal{N}(X)$,

$$
K \cap \operatorname{Conv} N \cap \operatorname{cl}_{X}\left(\bigcap_{x \in \operatorname{Conv} N} M(x)\right)=K \cap \operatorname{Conv} N \cap \bigcap_{x \in \operatorname{Conv} N} M(x) .
$$

Further (1) in (iii) follows from

$$
\operatorname{cl}_{X}\left(\bigcap_{x \in D} M(x)\right)=\bigcap_{x \in D} M(x)
$$

which follows from the "transfer-closedness" of the sets $M(x)$ :

$$
\bigcap_{x \in D} \operatorname{cl}_{X}(M(x))=\bigcap_{x \in D} M(x)
$$

which is used instead of (iii) by Ansari, Lin, and Yao [4].

(ii) replaces finite-dimensional subspaces used by Brèzis, Nirenberg, and Stampacchia [9] and in [26, 28] by polytopes, as it is done in [17]. Kalmoun [36] uses, instead, a transferclosed map on polytopes.

Finally, (i) is more general than the assumption of compactness of $\operatorname{cl}_{X}\left(\bigcap_{x \in N} M(x)\right)$ for some $N \in \mathcal{N}(X)$, which is used in [21]. This comes out in the following example.

Example 2.1 Let $X$ be an infinite-dimensional real Hilbert space endowed with norm $\|x\|$ and scalar product $\langle x, y\rangle$. For $x \in X$, define

$$
M(x)=\left\{y \in X:\langle y, x\rangle \leq\|x\|^{2}\right\} .
$$


Then $M(x)=X$ if $x=0$, and $M(x)$ is a closed convex halfspace of $X$ if $x \neq 0$. Furthermore, for all $x \in X$ and $r=\|x\|$, we have

$$
K_{r}=\{y \in X:\|y\| \leq r\} \subset M(x),
$$

since by the Cauchy-Schwarz inequality, for all $y \in K_{r}$,

$$
\langle y, x\rangle \leq|\langle y, x\rangle| \leq\|y\|\|x\| \leq\|x\|^{2} .
$$

This shows that $M$ is a KKM covering. Indeed, let $N \in \mathcal{N}(X)$. Define $\rho=\max \{\|x\|: x \in N\}$. Then Conv $N \subset K_{\rho}$ and $K_{\rho} \subset \bigcup\{M(x): x \in N\}$, and the claim follows.

Since $M(x)$ is weakly closed for all $x \in X$, (ii) and (iii) of Theorem 2.2 are satisfied. With $K_{1}$ convex and weakly compact, we next claim that $\bigcap\left\{M(x): x \in K_{1}\right\}=\{0\}$. Indeed, assume that there exists $y_{1} \in \bigcap\left\{M(x): x \in K_{1}\right\}, y_{1} \neq 0$. Then choose $x_{1}=\tau y_{1}$ with $0<\tau<1$ such that $x_{1} \in K_{1}$. Then $y_{1} \in M\left(x_{1}\right)$ leads to $\tau \geq 1$, which is absurd. Hence condition (i) is satisfied. Therefore Theorem 2.2 applies with respect to the weak topology.

On the contrary, we cannot find some $N \in \mathcal{N}(X)$ such that $\bigcap\{M(x): x \in N\}$ is compact. Indeed, consider the finite-dimensional subspace $F=\operatorname{span} N$. Since $X$ is of infinite dimension, there exists $z \neq 0$ such that $z \perp F$, and hence $\mathbb{R} z \subset \bigcap\{M(x): x \in N\}$.

Based on the FKKM Theorem 2.2, we establish the following general existence theorem for extended real-valued equilibria.

Theorem 2.3 Let $C$ be a closed convex subset of a real Hausdorff topological vector space $E$. Let the extended real-valued function $f: C \rightarrow(-\infty,+\infty], f \not \equiv \infty$, and a real-valued bifunction $\varphi: C \times C \rightarrow \mathbb{R}$, nonnegative on the diagonal of $C \times C$, be given such that the following assumptions are satisfied:

$\left(A_{1}\right)$ For all $x \in C, f+\varphi(x, \cdot)$ is quasiconvex on $C$.

$\left(A_{2}\right)$ There exist a compact subset $\tilde{K}$ of $E$ and $\tilde{z} \in C \cap \tilde{K}$ such that for all $x \in C \backslash \tilde{K}$,

$$
\varphi(x, \tilde{z})+f(\tilde{z})<f(x) .
$$

$\left(A_{3}\right)$ For all $y \in C$ and $N \in \mathcal{N}(C), f-\varphi(\cdot, y)$ is lower semicontinuous in Conv $N$.

$\left(A_{4}\right)$ For all $N \in \mathcal{N}(C)$ and every net $\left(x_{t}\right)_{t \in T} \subset C$ that converges to some $x \in \operatorname{Conv} N$, we have

$$
f\left(x_{t}\right) \leq \varphi\left(x_{t}, y\right)+f(y) \quad \forall y \in \operatorname{Conv} N, \forall t \in T
$$

implies

$$
f(x) \leq \varphi(x, y)+f(y) \quad \forall y \in \operatorname{Conv} N .
$$

Then there exists a solution $\hat{x}$ of the extended real-valued equilibrium problem $\mathcal{P}[\varphi, f ; C]$, that is, for all $y \in C$, we have

$$
\varphi(\hat{x}, y)+f(y) \geq f(\hat{x}) .
$$


Proof We define for any $z \in C=X$ the set

$$
M(z)=\{y \in C \mid f(y) \leq \varphi(y, z)+f(z)\} .
$$

To obtain the conclusion, we have to show that $M$ is a KKM covering that satisfies (i)-(iii).

Take $K_{0}=\{\tilde{z}\}$, which trivially is convex compact, and (i) holds by $\left(A_{2}\right)$.

(ii) follows from $\left(A_{3}\right)$.

(iii) is implied by $\left(A_{4}\right)$. Indeed, let $N \in \mathcal{N}(C)$. Then $K_{0} \cup N=\{\tilde{z}\} \cup N=: N^{\prime} \in \mathcal{N}(C)$ and $G=\operatorname{Conv}\left(K_{0} \cup N\right)=\operatorname{Conv} N^{\prime}$. Further, let $K$ be a compact in $C$, and let

$$
x^{\prime} \in G \cap \operatorname{cl}_{C}\left(\bigcap_{x \in G} M(x) \cap K\right) .
$$

This means that $x^{\prime}=\lim x_{t}$ for some net $\left\{x_{t}\right\}_{t \in T} \subset K$ and $x_{t}$ satisfy for all $t \in T$ and $x \in G$,

$$
f\left(x_{t}\right) \leq \varphi\left(x_{t}, x\right)+f(x) .
$$

By $\left(A_{4}\right)$, for all $x \in G$, we have

$$
f\left(x^{\prime}\right) \leq \varphi\left(x^{\prime}, x\right)+f(x)
$$

hence

$$
x^{\prime} \in G \cap\left(\bigcap_{x \in G} M(x) \cap K\right),
$$

and (iii) is proved.

Finally, we show that $M$ is a KKM covering by an indirect argument. Assume that there exist $z_{i} \in C$ and $\lambda_{i} \geq 0(i=1, \ldots, k)$ with $\sum \lambda_{i}=1$ such that

$$
\bar{z}:=\sum_{i=1}^{k} \lambda_{i} z_{i} \notin \cup_{i=1}^{k} M\left(z_{i}\right),
$$

that is, $\bar{z} \in C$ satisfies

$$
\varphi\left(\bar{z}, z_{i}\right)+f\left(z_{i}\right)<f(\bar{z}), \quad i=1, \ldots, k .
$$

By quasiconvexity and $\left(A_{1}\right)$ we arrive at $f(\bar{z})<\infty$ and

$$
\varphi(\bar{z}, \bar{z})+f(\bar{z})<f(\bar{z}), \quad i=1, \ldots, k,
$$

which contradicts $\varphi(\bar{z}, \bar{z}) \geq 0$. The theorem is proved.

\section{Existence of extended real-valued equilibria with bifunctions of monotone type}

As introduced by [26, 28] and independently in Aubin's monograph [5, Def. 2, Sect. 13.2], in analogy to pseudomonotone operators introduced and studied by Brèzis [8], a bifunction $\varphi: X \times X \rightarrow \mathbb{R}$ is called Brèzis pseudomonotone or topologically pseudomonotone 
(shortly p.m.) on a subset $X$ of $E$ if for any net $\left\{x_{t}\right\}_{t \in T}$ that converges to some $x \in X$ and satisfies $\liminf _{t \in T} \varphi\left(x_{t}, x\right) \geq 0$, we have for all $y \in X$,

$$
\varphi(x, y) \geq \limsup _{t \in T} \varphi\left(x_{t}, y\right)
$$

Note that $\varphi(x, y):=s(y)-s(x)$ with a lower semicontinuous (l.s.c.) function $s: C \rightarrow \mathbb{R}$ is a simple example of a p.m. bifunction.

The following proposition shows that such bifunctions $\varphi$ satisfy the assumption $\left(A_{4}\right)$, provided that the function $f$ is l.s.c.

Proposition 3.1 Let $C$ be a closed convex subset of a real Hausdorff topological vector space. Let an extended real-valued function $f: C \rightarrow(-\infty,+\infty], f \not \equiv \infty$ be l.s.c., and let a real-valued bifunction $\varphi: C \times C \rightarrow \mathbb{R}$ be p.m. Then the pair $[\varphi, f]$ satisfies assumption $\left(A_{4}\right)$.

Proof Let $N \in \mathcal{N}(C)$, and let $\left(x_{t}\right)_{t \in T}$ be a net that converges to some $x \in \operatorname{Conv} N$ such that

$$
f\left(x_{t}\right) \leq \varphi\left(x_{t}, y\right)+f(y) \quad \forall y \in \operatorname{Conv} N, \forall t \in T .
$$

Choosing $y=x \in \operatorname{Conv} N$, by the lower semicontinuity of $f$ we obtain

$$
\liminf _{t \in T} \varphi\left(x_{t}, x\right) \geq \liminf _{t \in T}\left[f\left(x_{t}\right)-f(x)\right] \geq 0 .
$$

Hence, for all $y \in \operatorname{Conv} N \subset C$, we get

$$
\begin{aligned}
\varphi(x, y) & \geq \limsup _{t \in T} \varphi\left(x_{t}, y\right) \geq \limsup _{t \in T}\left[f\left(x_{t}\right)-f(y)\right] \\
& \geq \liminf _{t \in T}\left[f\left(x_{t}\right)-f(y)\right] \geq f(x)-f(y),
\end{aligned}
$$

since $\varphi$ is p.m. and $f$ is l.s.c., and the proposition follows.

Thus we immediately obtain the following existence result for extended real-valued equilibria with p.m. bifunctions.

Theorem 3.1 Let $C$ be a closed convex subset of a real Hausdorff topological vector space $E$. Let an extended real-valued l.s.c. function $f: C \rightarrow(-\infty,+\infty], f \not \equiv \infty$, and a real-valued p.m. bifunction $\varphi: C \times C \rightarrow \mathbb{R}$, nonnegative on the diagonal of $C \times C$, be given such that the following assumptions are satisfied:

$\left(B_{1}\right)$ For all $x \in C, f+\varphi(x, \cdot)$ is quasiconvex on $C$.

$\left(B_{2}\right)$ There exist a compact subset $\tilde{K}$ of $E$ and $\tilde{z} \in C \cap \tilde{K}$ such that for all $x \in C \backslash \tilde{K}$,

$$
\varphi(x, \tilde{z})+f(\tilde{z})<f(x)
$$

$\left(B_{3}\right)$ For all $y \in C$ and $N \in \mathcal{N}(C), \varphi(\cdot, y)$ is upper semicontinuous on Conv $N$.

Then there exists a solution $\hat{x} \in C \cap \tilde{K}$ of the extended real-valued equilibrium problem $\mathcal{P}[\varphi, f ; C]$, that is, for all $y \in C$,

$$
\varphi(\hat{x}, y)+f(y) \geq f(\hat{x}) .
$$


Next, we sharpen some assumptions in Theorem 3.1 and derive the following corollary.

Corollary 3.1 [26, Satz 1.6] Let C be a closed convex subset of a real Hausdorff topological vector space $E$. Let an extended real-valued convex l.s.c. function $f: C \rightarrow(-\infty,+\infty], f \not \equiv$ $\infty$, and a real-valued p.m. bifunction $\varphi: C \times C \rightarrow \mathbb{R}$, nonnegative on the diagonal of $C \times C$, be given such that the following assumptions are satisfied:

$\left(C_{1}\right)$ For all $x \in C, \varphi(x, \cdot)$ is convex on $C$.

$\left(C_{2}\right)$ There exist a compact subset $\tilde{K}$ of $E$ and $\tilde{z} \in C \cap \tilde{K}$ such that for all $x \in C \backslash \tilde{K}$,

$$
\varphi(x, \tilde{z})+f(\tilde{z})<f(x)
$$

$\left(C_{3}\right)$ For all $y \in C$ and $F \in \mathcal{F}(C), \varphi(\cdot, y)$ is upper semicontinuous on $C \cap F$.

Then there exists a solution $\hat{x} \in C \cap \tilde{K}$ of the extended real-valued equilibrium problem $\mathcal{P}[\varphi, f ; C]$.

Proof We only have to show that (C3) implies (B3). For that, let $N \in \mathcal{N}(C), N=\left\{z_{j}: 1 \leq\right.$ $j \leq k\}$. Define $F=\operatorname{span}\left\{z_{j}: 1 \leq j \leq k\right\}$. Then $F \in \mathcal{F}(C)$ and Conv $N \subset C \cap F$. Hence if $f$ is l.s.c. on $C \cap F$, then $f$ is l.s.c. on $\operatorname{Conv} N$.

Note that in the case $C=E$, conditions (C3) and (B3) are in fact equivalent. Indeed, for all $x_{0} \in F$ and $F \in \mathcal{F}(E)$, we can construct some $N \in \mathcal{N}(F)$ such that $x_{0}$ is an interior point of Conv $N$. Hence the continuity on Conv $N, N \in \mathcal{N}(E)$, implies the continuity on $F$ for all $F \in \mathcal{F}(E)$.

From now on we impose that $\varphi$ vanishes on the diagonal of $C \times C$ and that for $x \in$ $C, \varphi(x, \cdot)$ is convex. Let us recall from $[7,26]$ that a bifunction $\varphi: C \times C \rightarrow \mathbb{R}$ is called monotone on the closed convex subset $C$ of $E$ if for all $x, y \in C$,

$$
\varphi(x, y)+\varphi(y, x) \leq 0
$$

Note that $\varphi(x, y):=c(y)-c(x)$ with $c: C \rightarrow \mathbb{R}$ is a simple example of a monotone bifunction. For a monotone bifunction $\varphi$, the following relaxed notion of continuity is important: $\varphi$ is called hemicontinuous on $C$ if for all $x, y \in C$, the function $t \in[0,1] \rightarrow \varphi(\operatorname{ty}+(1-t) x, y)$ is upper semicontinuous at $t=0$. For a hemicontinuous monotone bifunction $\varphi$, the "Minty trick" [10, Lemma 1], [42, Lemma 1] applies:

Proposition 3.2 Let $\varphi: C \times C \rightarrow \mathbb{R}$, let $f: C \rightarrow(-\infty,+\infty], f \not \equiv \infty$, be convex and l.s.c., and let $D$ be a convex subset of $C$. Consider the statements

(i) $\quad x \in D, \varphi(x, y)+f(y)-f(x) \geq 0 \quad \forall y \in D$;

(ii) $x \in D, \varphi(y, x)+f(x)-f(y) \leq 0 \quad \forall y \in D$.

If $\varphi$ is monotone on $D$, then (i) implies (ii); if $\varphi$ is hemicontinuous on $D$, then (i) follows from (ii). 
Proof (i) $\Rightarrow$ (ii) is obvious for monotone $\varphi$. - To show (ii) $\Rightarrow$ (i), let $y \in D$. Then $z_{s}=x+$ $s(y-x) \in D$ for $s \in(0,1]$, and hence $\varphi\left(z_{s}, x\right) \leq f\left(z_{s}\right)-f(x)$. Since $\varphi\left(z_{s}, \cdot\right)$ and $f$ are convex,

$$
\begin{aligned}
0 & =\varphi\left(z_{s}, z_{s}\right) \leq s \varphi\left(z_{s}, y\right)+(1-s)\left[f\left(z_{s}\right)-f(x)\right] \\
& \leq s\left[\varphi\left(z_{s}, y\right)-f\left(z_{s}\right)\right]+s f(y),
\end{aligned}
$$

and thus $-f(y) \leq \varphi\left(z_{s}, y\right)-f\left(z_{s}\right)$. Letting $s \rightarrow 0$ results in $-f(y) \leq \varphi(x, y)-f(x)$, since $\varphi$ is hemicontinuous and $f$ is l.s.c.

Now we can show that a hemicontinuous monotone bifunction $\varphi$ that is l.s.c. in the second argument together with a convex l.s.c. extended real-valued function $f$ satisfies assumption $\left(A_{4}\right)$ of the general existence Theorem 2.3: Let $D$ be an arbitrary convex subset of $C$, and let $\left\{x_{t}\right\} \subset C$ be a net converging to some $x \in D$ such that $\varphi\left(x_{t}, z\right) \geq f\left(x_{t}\right)-f(z)$ for all $z \in D$ and $t \in T$. Since $\varphi$ is monotone, $\varphi\left(z, x_{t}\right) \leq f(z)-f\left(x_{t}\right)$ for all $z \in D$ and $t \in T$. Since $\varphi(z, \cdot)$ and $f$ are l.s.c., we get that $\varphi(z, x) \leq f(z)-f(x)$ for all $z \in D$. By Proposition 3.2 we conclude that $\varphi(x, \cdot) \geq f(x)-f(\cdot)$ on $D$, as claimed.

Thus Theorem 2.3 applies to conclude the existence of equilibria with monotone bifunctions. However, its assumption $\left(A_{3}\right)$ imposes for any $y \in C$ the upper semicontinuity of $\varphi(\cdot, y)$ on finite-dimensional convex parts $\operatorname{Conv} N$ for $N \in \mathcal{N}(C)$, which is slightly stronger than the hemicontinuity. To arrive at an existence result for extended real-valued equilibria with monotone bifunctions that are only hemicontinuous, we again appeal to the basic Theorem 2.2 and slightly modify the monotonicity argument of [7, Lemma 2].

Theorem 3.2 [26, Satz 1.8] Let C be a closed convex subset of a real Hausdorff topological vector space. Let an extended real-valued convex l.s.c. function $f: C \rightarrow(-\infty,+\infty], f \not \equiv \infty$, and a real-valued hemicontinuous monotone bifunction $\varphi: C \times C \rightarrow \mathbb{R}$ that vanishes on the diagonal of $C \times C$ be given such that the following assumptions are satisfied:

$\left(D_{1}\right)$ For all $x \in C, \varphi(x, \cdot)$ is convex and l.s.c. on $C$.

$\left(D_{2}\right)$ There exist a compact subset $\tilde{K}$ of $E$ and $\tilde{z} \in C \cap \tilde{K}$ such that for all $x \in C \backslash \tilde{K}$,

$$
\varphi(x, \tilde{z})+f(\tilde{z})<f(x) .
$$

Then there exists a solution $\hat{x}$ of the extended real-valued equilibrium problem $\mathcal{P}[\varphi, f ; C]$.

Proof In virtue of Proposition 3.2, we have to show the existence of $\hat{x} \in C \cap K$ that satisfies

$$
\varphi(y, \hat{x}) \leq f(y)-f(\hat{x})
$$

for all $y \in C$. Thus we apply Theorem 2.2 to the subsets

$$
M(y)=\{x \in C \mid \varphi(y, x) \leq f(y)-f(x)\}, \quad y \in C,
$$

of $X=C$. Since $K_{0}=\{\tilde{z}\}$ is convex compact and $M(\tilde{z})$ is relatively compact by $\left(D_{2}\right)$, (i) is satisfied. The continuity assumptions on $f$ and $\varphi(y, \cdot)$ by $\left(D_{1}\right)$ ensure that $M(y)$ is closed for all $y \in C$. Hence (ii) and (iii) hold. It remains to show that $M: C \rightarrow 2^{C}$ is a KKM map. Let 
$\left\{y_{1}, \ldots, y_{k}\right\}$ be an arbitrary finite subset of $C$, and let $\left\{\lambda_{1}, \ldots, \lambda_{k}\right\}$ be arbitrary nonnegative reals with $\sum_{i=1}^{k} \lambda_{i}=1$. Suppose that

$$
y^{*}=\sum_{i=1}^{k} \lambda_{i} y_{i} \notin \bigcup_{i=1}^{k} M\left(y_{i}\right)
$$

This means that

$$
\varphi\left(y_{i}, y^{*}\right)+f\left(y^{*}\right)>f\left(y_{i}\right), \quad \forall i=1, \ldots, k .
$$

On the other hand, since $\varphi\left(y_{i}, \cdot\right)$ and $f$ are convex,

$$
\varphi\left(y_{i}, y^{*}\right)+f\left(y^{*}\right) \leq \sum_{j=1}^{k} \lambda_{j}\left[\varphi\left(y_{i}, y_{j}\right)+f\left(y_{j}\right)\right] \quad \forall i=1, \ldots, k .
$$

Hence altogether by linear combination with $\lambda_{i} \geq 0$ we have

$$
\sum_{i=1}^{k} \sum_{j=1}^{k} \lambda_{i} \lambda_{j} \varphi\left(y_{i}, y_{j}\right)+\sum_{j=1}^{k} \lambda_{j} f\left(y_{j}\right)>\sum_{i=1}^{k} \lambda_{i} f\left(y_{i}\right) .
$$

This results in

$$
2 \sum_{i=1}^{k} \sum_{j=1}^{k} \lambda_{i} \lambda_{j} \varphi\left(y_{i}, y_{j}\right)=\sum_{i=1}^{k} \sum_{j=1}^{k} \lambda_{i} \lambda_{j}\left[\varphi\left(y_{i}, y_{j}\right)+\varphi\left(y_{j}, y_{i}\right)\right]>0,
$$

which contradicts the monotonicity of $\varphi$. Hence there exists $\hat{z} \in C$ that is an extended real-valued equilibrium of $\mathcal{P}[\varphi, f ; C]$. In view of $\left(D_{2}\right), \hat{z} \in \tilde{K}$.

\section{Variational inequalities of monotone type with set-valued operators. An equilibrium approach}

In this section, we derive existence results for variational inequalities (VIs) of monotone type from our existence results for extended real-valued equilibria. Here we focus to variational inequalities of the first kind with set-valued operators ("multis") of monotone type in general topological vector spaces (t.v.s.).

The crucial element in our approach to existence results for multis is the following extension lemma [29, Theorem 2.2], which is a refined version of the famous Fan-GlicksbergHoffman theorem of alternative, which can be derived from Kneser's minimax theorem [37] and from Simons' more general two-function minimax theorem [55, Theorem 5] or can be directly proved from the separation theorem [7, 29].

Lemma 4.1 Let $C$ be a convex set in a vector space. Llet $K$ be a compact convex set in a topological vector space. Let $L$ be a real-valued functional on $C \times K$ such that, for all $y \in K$, $L(\cdot, y)$ is convex and, for every $x \in C, L(x, \cdot)$ is concave and upper semicontinuous. Then the following statements are equivalent.

1. For each $x \in C$, there exists $y \in K$ such that $L(x, y) \geq 0$.

2. There exists $\tilde{y} \in K$ such that $L(x, \tilde{y}) \geq 0$ for each $x \in C$. 
Moreover, we need the well-known Berge theorem for maximum functions with setvalued maps; see [6, Theorem 2, Chap. 6, p. 122]. For that, we recall (see [6, Chap. 6]) that a set-valued map $S: X \rightarrow 2^{Y}$ in topological spaces $X, Y$ is upper semicontinuous at $x_{0} \in X$ if for any open set $O \supset S\left(x_{0}\right)$ there is a neighborhood $U$ of $x_{0}$ such that $O \supset S(x)$ for any $x \in U$; $S$ is upper semicontinuous (u.s.c.), if $S$ is upper semicontinuous at any $x_{0} \in X$.

Lemma 4.2 Let $X$ and $Y$ be topological spaces, let $f: X \times Y \rightarrow \mathbb{R}$, let $S: X \rightarrow 2^{Y}$ be a setvalued map, and let

$$
\bar{f}(x)=\sup _{y \in S(x)} f(x, y) \quad \forall x \in X
$$

Suppose that $f$ is upper semicontinuous and $S$ is upper semicontinuous with compact values. Then $\bar{f}$ is upper semicontinuous.

Let $E$ be real Hausdorff topological vector space with dual space $E^{*}$ and duality $\langle\cdot, \cdot\rangle$. Further, as before, let $C$ be a closed convex nonempty subset of $E$. Let $S: C \rightarrow 2^{E^{*}}$ be a set-valued operator with nonempty, convex, and $\sigma\left(E^{*}, E\right)$-compact values $S(x)$ for $x \in C$.

We are interested in the variational inequality problem $\mathcal{P}(S ; C)$ : Find a pair $(\hat{x}, \hat{\xi}) \in E \times E^{*}$ such that $\hat{x} \in C, \hat{\xi} \in S(\hat{x})$, and for all $y \in C$,

$$
\langle\hat{\xi}, y-x\rangle \geq 0
$$

To apply our theory for equilibria of the previous section, we consider the real-valued bifunction $\varphi: C \times C \rightarrow \mathbb{R}$,

$$
\varphi(x, y):=\max _{\xi \in S(x)}\langle\xi, y-x\rangle
$$

which clearly vanishes on the diagonal of $C \times C$ and is convex and l.s.c. on $C$ with respect to the second argument.

Now we can derive from Theorem 3.2 the following existence result for VIs with monotone set-valued operators.

Theorem 4.1 Let $C$ be a closed convex subset of a real Hausdorff topological vector space $E$. Let $S: C \rightarrow 2^{E^{*}}$ be a set-valued hemicontinuous operator, that is, for all $x, y \in C, t \in[0,1] \rightarrow$ $S(\mathrm{tx}+(1-t) y)$ is upper semicontinuous at $t=0$ and is monotone, that is, for all $x, y \in C$, $\xi \in S(x)$, and $\eta \in S(y)$, we have $\langle\xi-\eta, x-y\rangle \geq 0$. Suppose there exist a compact subset $\tilde{K}$ of $E$ and $\tilde{z} \in C \cap \tilde{K}$ such that for all $x \in C \backslash \tilde{K}$, there exist $\zeta \in S(\tilde{z})$ such that

$$
\langle\zeta, x-\tilde{z}\rangle<0
$$

Then there exists a solution $\hat{x}$ to the variational inequality problem $\mathcal{P}(S ; C)$.

Proof Obviously, the bifunction $\varphi$ given by (2) is monotone and hemicontinuous by Lemma 4.2. Since the coercivity condition $\left(D_{2}\right)$ is satisfied, Theorem 3.2 yields the existence of $\hat{x} \in C$ such that for each $y \in C$, there exists $\xi \in S(\hat{x})$ such that $\langle\xi, y-x\rangle \geq 0$. Finally, apply Lemma 4.1 to obtain the result. 
Next, let $S: C \rightarrow 2^{E^{*}}$ be a set-valued operator with nonempty, convex, and $\sigma\left(E^{*}, E\right)$ compact values $E(x)$ for $x \in C$ that is u.s.c. from every finite-dimensional part $C \cap F$ (where $F$ is a finite-dimensional subspace of $E$ ) into $E^{*}$ equipped with the weak topology $\sigma\left(E^{*}, E\right)$. Then $S$ is called pseudomonotone (p.m.) (see, e.g., [52, Definition 2.10.1], going back to [8]) if the following condition holds:

$$
\begin{aligned}
& \text { If a net }\left\{x_{t}\right\}_{t \in T} \subset C \text { converges to } x \in C \text { and } \xi_{t} \in S\left(x_{t}\right) \text { satisfies } \limsup _{t \in T}\left\langle\xi_{t}, x-x_{t}\right\rangle \geq 0 \text {, } \\
& \text { then for each } y \in C \text {, there is } \xi \in S(x) \text { such that }\langle\xi, y-x\rangle \geq \limsup _{t \in T}\left\langle\xi_{t}, y-x_{t}\right\rangle .
\end{aligned}
$$

Here we focus on this notion of generalized monotonicity. For other notions of generalized monotonicity for multis and their relation to topological pseudomonotonicity, we refer to [52, Sect. 2.10].

Again, we use construction (2). It is evident that a p.m. set-valued operator gives a p.m. bifunction $\varphi$. So by a similar reasoning as before, in particular, using Lemmas 4.1 and 4.2, we can conclude from Theorem 3.1 the following existence result for VIs with p.m. set-valued operators.

Theorem 4.2 Let $C$ be a closed convex subset of a real Hausdorff topological vector space $E$. Let $S: C \rightarrow E^{*}$ be a p.m. set-valued operator that is u.s.c. from every part $C \cap F$ into $E^{*}$ for any finite-dimensional subspace $F$ of $E$. Suppose there exist a compact subset $\tilde{K}$ of $E$ and $\tilde{z} \in C \cap \tilde{K}$ such that for all $x \in C \backslash \tilde{K}$, there exists $\zeta \in S(\tilde{z})$ such that $\langle\zeta, x-\tilde{z}\rangle<0$. Then there exists a solution $\hat{x}$ to the variational inequality problem $\mathcal{P}(S ; C)$.

\section{Extended real-valued equilibrium problems of monotone type in a reflexive Banach space}

Let $V$ be a reflexive Banach space with dual $V^{*}$. We denote by $\langle\cdot, \cdot\rangle$ the duality pairing between $V$ and $V^{*}$ and by $\|\cdot\|$ and $\|\cdot\|_{*}$ the norm and the dual norm on $V$ and $V^{*}$, respectively. We denote by " $\rightarrow$ " and " $\rightarrow$ " the weak and strong convergence in $V$, respectively. As before, $C$ stands for a nonempty closed convex subset of $V$.

In the following, we use weak sequential convergence on $V$ and, in particular, the compactness of the unit ball of $V$ with respect to weak sequential convergence. Moreover, since from any weakly convergent net in a reflexive Banach space there can be extracted a weakly convergent subsequence (with the same limit) (see [11, Prop. 7.2]), we can simplify the definition of a p.m. bifunction $\varphi$ as follows. Now $\varphi: C \times C \rightarrow \mathbb{R}$ is called Brèzis pseudomonotone or topologically pseudomonotone (p.m.) on $C$ if for any sequence $\left\{x_{n}\right\}_{n \in \mathbb{N}} \subset C$ that converges weakly to some $x \in V$ and satisfies $\liminf _{n \in \mathbb{N}} \varphi\left(x_{n}, x\right) \geq 0$, we have

$$
\varphi(x, y) \geq \limsup _{n \in \mathbb{N}} \varphi\left(x_{n}, y\right)
$$

for all $y \in C$. The stability of monotonicity with respect to addition is evident, and it can be shown that the sum of two p.m. bifunctions is p.m., too; see [30, Proposition].

Also, for further use, we note that a hemicontinuous monotone operator $T$ defined throughout $V$ is continuous on finite-dimensional subspaces and pseudomonotone on $V$; see [8, Propositions 6 and 9] and [59, Proposition 27.6]. Hence, in particular, the bifunction $\varphi_{T}(x, y):=\langle\mathrm{Tx}, y-x\rangle$ associated with $T$ is p.m. on $V$. 
Now we are in a position to apply our general existence theory of Sect. 3 and derive existence results for extended real-valued equilibrium problems of monotone type in the setting of a reflexive Banach space, first, on bounded sets and then on unbounded sets, where we discuss the issue of coercivity following [26].

\subsection{Extended real-valued equilibrium problems of monotone type on bounded sets}

Let $V$ be a reflexive Banach space with norm $\|\cdot\|$ and dual $V^{*}$. As before, $C$ stands for a nonempty closed convex subset of $V$. In the following, let an extended real-valued function $f: C \rightarrow(-\infty,+\infty], f \not \equiv \infty$, be convex and l.s.c., and let a real-valued bifunction $\varphi: C \times C \rightarrow \mathbb{R}$ vanish on the diagonal of $C \times C$ and be convex and l.s.c. with respect to the second argument. We are interested in the existence of a solution $\hat{x}$ of the extended real-valued equilibrium problem $\mathcal{P}[\varphi, f ; C]$, that is,

$$
\varphi(\hat{x}, y)+f(y) \geq f(\hat{x})
$$

for all $y \in C$. From Theorem 3.1 we immediately obtain the following:

Corollary 5.1 Let, in addition, $C$ be bounded in the reflexive Banach space $V$, and let a p.m. bifunction $\varphi: C \times C \rightarrow \mathbb{R}$ be such that for all $y \in C$ and $N \in \mathcal{N}(C), \varphi(\cdot, y)$ is upper semicontinuous on Conv $N$. Then there exists a solution $\hat{x} \in C \cap \tilde{K}$ of the extended realvalued equilibrium problem $\mathcal{P}[\varphi, f ; C]$.

This result can be compared with the recent existence result in [14, Theorem 2.1], where an additional maximal monotone bifunction appears, and instead of $\mathcal{P}[\varphi, f ; C]$, a regularized equilibrium problem (with $f=0$ ) with the duality map as a regularizer is considered.

When the bifunction $\varphi$ is only defined on $C \times C$, where $C$ is a proper subset of $V$, we can appeal to Theorem 3.2 and directly obtain the following Hartman-Stampacchia-like result [34].

Corollary 5.2 Let, in addition, $C$ be bounded in the reflexive Banach space $V$, and let $\varphi: C \times C \rightarrow \mathbb{R}$ be a hemicontinuous and monotone bifunction. Then there exists a solution $\hat{x}$ of the extended real-valued equilibrium problem $\mathcal{P}[\varphi, f ; C]$.

\subsection{Extended real-valued equilibrium problems of monotone type on unbounded sets. Coercivity}

Throughout this subsection, we assume that the equilibrium problem $\mathcal{P}[\varphi, f ; D]$ is solvable for any bounded closed convex subset $D \subset C$. For brevity, we write $\psi(x, y):=\varphi(x, y)+f(y)-$ $f(x)$.

First, we consider the coercivity condition that goes back to Moré [43, Theorem 2] dealing with the solution of complementarity problems.

Proposition 5.1 In addition, assume tha

$$
\varphi(x, \tilde{z})+f(\tilde{z}) \leq f(x), \quad \forall x \in C \text { with }\|x\|=\rho,
$$

for some $\tilde{z} \in C$ with $\rho>\|\tilde{z}\|$. Then there exists a solution of $\mathcal{P}[\varphi, f ; C]$ in $K(0, \rho):=\{x \in V$ : $\|x\| \leq \rho\}$. 
Proof By assumption there exists a solution $\bar{x}$ of $\mathcal{P}\left[\varphi, f ; C_{\rho}\right]$, where $C_{\rho}:=C \cap K(0, \rho)$. We distinguish two cases.

Suppose $\|\bar{x}\|<\rho$. Then choose $x_{1} \in C$ with $\left\|x_{1}\right\|>\rho$. Take $x_{2}=\tau x_{1}+(1-\tau) \bar{x}$. For sufficiently small $\tau>0$, we have $x_{2} \in C_{\rho}$, and hence

$$
0 \leq \psi\left(\bar{x}, x_{2}\right) \leq \tau \psi\left(\bar{x}, x_{1}\right)
$$

and thus $\psi\left(\bar{x}, x_{1}\right) \geq 0$.

Otherwise, $\|\bar{x}\|=\rho$, and hence $\psi(\bar{x}, \tilde{z})=0$. For an arbitrary $x_{1} \in C$ with $\left\|x_{1}\right\|>\rho$, take $x_{2}=\tau x_{1}+(1-\tau) \tilde{z}$. For sufficiently small $\tau>0$, we have $x_{2} \in C_{\rho}$ and hence obtain as above $\psi\left(\bar{x}, x_{1}\right) \geq 0$. So in both cases, $\bar{x}$ solves $\mathcal{P}[\varphi, f ; C]$.

Next, we consider the coercivity condition that goes back to Stampacchia in [58, Theorem 2.5].

\section{Proposition 5.2}

A necessary and sufficient condition for the solvability of $\mathcal{P}[\varphi, f ; C]$ is the existence of a real number $\rho>0$ such that a solution $x_{\rho}$ of $\mathcal{P}\left[\varphi, f ; C_{\rho}\right]$ with $C_{\rho}=C \cap K(0, \rho)$ satisfies $\left\|x_{\rho}\right\|<\rho$.

Proof Necessity. Just choose $\rho>\|\bar{x}\|$ for a solution $\bar{x}$ of $\mathcal{P}[\varphi, f ; C]$.

Sufficiency. Let $x_{\rho}$ solve $\mathcal{P}\left[\varphi, f ; C_{\rho}\right]$ with $\left\|x_{\rho}\right\|<\rho$. To show that $x_{\rho}$ solves $\mathcal{P}[\varphi, f ; C]$, let $y \in C \backslash K(0, \rho)$ be arbitrary. Then $z=\tau y+(1-\tau) x_{\rho} \in C$ for $\tau \in(0,1)$, and for sufficiently small $\tau>0$, we have

$$
\|z\| \leq\left\|x_{\rho}\right\|+\tau\left(\|y\|-\left\|x_{\rho}\right\|\right) \leq \rho .
$$

Hence $0 \leq \psi\left(x_{\rho}, z\right) \leq \tau \psi\left(x_{\rho}, y\right)$, and thus $\psi\left(x_{\rho}, y\right) \geq 0$. The conclusion follows.

In addition, assume that $\varphi$ is monotone. Then the coercivity condition of Proposition 5.2 implies the existence of $x_{\rho}$ with $\left\|x_{\rho}\right\|<\rho$ such that $\psi\left(y, x_{\rho}\right) \leq 0$ for all $y \in C_{\rho}$. Hence $x_{\rho}$ satisfies the coercivity condition of Proposition 5.1.

Next, we provide asymptotic coercivity conditions for equilibrium problems on unbounded sets.

Theorem 5.1 Let $\varphi: C \times C \rightarrow \mathbb{R}$ be p.m. or hemicontinous and monotone. Suppose there exists $x_{0} \in C$ such that

$$
\limsup _{\|x\| \rightarrow \infty, x \in C}\left[\varphi\left(x, x_{0}\right)-f(x)\right]<-f\left(x_{0}\right)
$$

Then there exists a solution to $\mathcal{P}[\varphi, f ; C]$.

Proof By assumption there exists a solution $x_{n}$ of $\mathcal{P}\left[\varphi, f ; C_{n}\right]$ on the bounded set $C_{n}$ := $C \cap K(0, n)$ for all $n \in \mathbb{N}$. By a contradiction argument we can show that (3) implies that the norms $\left\|x_{n}\right\|$ are bounded. Hence there exists a weakly convergent subsequence $\left\{x_{k}\right\}_{k \in K}$, $K \subset \mathbb{N}$, such that $x_{k} \rightarrow \bar{x}$ for some $\bar{x} \in C$ and $\bar{x} \in C_{k}$ for sufficiently large $k \in K$. 
For a p.m. bifunction $\varphi$, we conclude from

$$
\liminf _{k \rightarrow \infty, k \in K} \varphi\left(x_{k}, \bar{x}\right) \geq \liminf _{k \rightarrow \infty, k \in K}\left[f\left(x_{k}\right)-f(\bar{x})\right] \geq 0
$$

that

$$
\begin{aligned}
\varphi(\bar{x}, y) & \geq \limsup _{k \rightarrow \infty, k \in K} \varphi\left(x_{k}, y\right) \\
& \geq \liminf _{k \rightarrow \infty, k \in K}\left[f\left(x_{k}\right)-f(y)\right] \\
& \geq f(\bar{x})-f(y) \quad \forall y \in C .
\end{aligned}
$$

Hence $\bar{x}$ solves $\mathcal{P}[\varphi, f ; C]$.

For a hemicontinous and monotone bifunction $\varphi$, we use the Minty trick as follows. By construction and Proposition 3.2 we have

$$
x_{k} \in C_{k}, \varphi\left(y, x_{k}\right) \leq f(y)-f\left(x_{k}\right) \quad \forall y \in C_{k} .
$$

Since the sets $C_{k}$ give rise to an ascending set sequence and exhaust the set $C$, it follows for all $y \in C$,

$$
\varphi(y, \bar{x}) \leq \liminf _{k \rightarrow \infty, k \in K, k \geq k_{0}(y)} \varphi\left(y, x_{k}\right) \leq \liminf _{k \rightarrow \infty, k \in K, k \geq k_{0}(y)}\left[f(y)-f\left(x_{k}\right)\right] \leq f(y)-f(\bar{x}) .
$$

By Proposition $3.2 \bar{x}$ solves $\mathcal{P}[\varphi, f ; C]$.

The asymptotic coercivity condition (3) is in particular satisfied if there is $x_{0} \in C$ such that

$$
\frac{f(x)-\varphi\left(x, x_{0}\right)}{\left\|x-x_{0}\right\|} \rightarrow \infty \quad \text { for } x \in C,\|x\| \rightarrow \infty .
$$

A coercivity condition of this type can already be found in [10, Theorem 3].

\subsection{Extended real-valued equilibrium problems of monotone type under asymptotic coercivity condition}

Under the asymptotic condition (4), we formulate the following useful existence results. First, in the monotone case, we combine Theorem 5.1 and Corollary 5.2 to immediately obtain the following:

Theorem 5.2 Let $C$ be a closed convex subset of the reflexive Banach space $V$. Let an extended real-valued function $f: C \rightarrow(-\infty,+\infty], f \neq \infty$, be convex and l.s.c. and let a realvalued bifunction $\varphi: C \times C \rightarrow \mathbb{R}$ vanish on the diagonal of $C \times C$ and be convex and l.s.c. with respect to the second argument. Further, let $\varphi: C \times C \rightarrow \mathbb{R}$ be hemicontinuous and monotone. Suppose the coercivity condition (4). Then there exists a solution $\hat{x}$ of the extended real-valued equilibrium problem $\mathcal{P}[\varphi, f ; C]$.

Finally, in this subsection, we treat the pseudomonotone case and formulate the following useful existence result, where part (A) extends and improves [27, Theorem 1], [28, 
Theorem 3], and [31, Theorem 2.3] by relaxing the upper semicontinuity condition on finite-dimensional parts to upper semicontinuity on convex hulls of finite subsets of $C$. In part (B), we introduce an applicable growth condition for the bifunction $\varphi$, which can be guaranteed in the context of variational-hemivariational inequalities we will study in the next section.

Theorem 5.3 (A) Let $V$ be a reflexive Banach space. Let the extended real-valued function $f: C \rightarrow(-\infty,+\infty], f \not \equiv \infty$, be convex and l.s.c. on the convex closed subset $C \subset V$. Let $\varphi: C \times C \rightarrow \mathbb{R}$ be a bifunction such that for all $x \in C, \varphi(x, x) \geq 0$ and $\varphi(x, \cdot)$ is convex and l.s.c. Moreover, suppose that $\varphi$ is p.m., $\varphi(\cdot, y)$ is upper semicontinuous on the convex hull of any finite subset of $C$ for all $y \in C$, and (4) holds. Then there exists a solution of the extended real-valued equilibrium problem $\mathcal{P}[\varphi, f ; C]$.

(B) Let, in addition, $A: V \rightarrow V^{*}$ be a hemicontinuous operator that satisfies

$$
\langle A(x)-A(y), x-y\rangle \geq\|x-y\| G(\|x-y\|) \quad \forall x, y \in V
$$

where the function $G: \mathbb{R}_{+}=[0,+\infty) \rightarrow \mathbb{R}_{+}$is strictly increasing with $G(0)=0$. Further, suppose that $\varphi$ satisfies, instead of (4), the following growth condition: there exists a positive constant $c_{1}$ such that

$$
\varphi(y, 0) \leq c_{1}(1+\|y\|) \quad \forall y \in C .
$$

Then there exists a solution $\hat{x}$ of the extended real-valued equilibrium problem $\mathcal{P}[A, \varphi, f ; C]$, that is, for all $y \in C$,

$$
\langle A(\hat{x}), y-\hat{x}\rangle+\varphi(\hat{x}, y)+f(y) \geq f(\hat{x}) .
$$

\section{Proof}

(A) This is a consequence of Theorem 3.1 in combination with Theorem 5.1.

(B) First, observe that the hemicontinuous and monotone operator $A$ defined throughout the space $V$ is pseudomonotone and upper semicontinuous on finite-dimensional subspaces. Hence the associated bifunction $\varphi_{A}(x, y)=\langle\operatorname{Ax}, y-x\rangle$ is p.m., and $\varphi_{A}(\cdot, y)$ is upper semicontinuous on the convex hull of any finite subset of $C$ for all $y \in C$; see the proof of Corollary 3.1.

Since summation preserves pseudomonotonicity (see [30]), the bifunction $\psi:=\varphi_{A}+\varphi$ is p.m., and $\psi(\cdot, y)$ is upper semicontinuous on the convex hull of any finite subset of $C$ for all $y \in C$.

We claim that $f(x)-\psi(x, 0), x \in C$, satisfies the asymptotic coercivity condition (4). Indeed, (5) and (6) entail the estimate

$$
-\psi(x, 0) \geq\|x\| G(\|x\|)+\langle A(0), x\rangle-c_{1}(1+\|x\|) \quad \forall x \in C,
$$

and $f$ is bounded below,

$$
f(x) \geq \lambda(x)+c_{f} \quad \forall x \in C
$$


with appropriate $\lambda \in V^{*}$ and $c_{f} \in \mathbb{R}$. This latter estimate can be obtained by strong separation (Hahn-Banach theorem) of the convex closed set epi $f=\{(x, r) \in C \times \mathbb{R}: r \geq f(x)\}$ from the compact set $\left(x_{1}, f\left(x_{1}\right)-1\right)$, where $x_{1} \in C$ is taken such that $f\left(x_{1}\right)<\infty$.

Thus we can again apply the Theorem 3.1 in combination with Theorem 5.1 to obtain the conclusion.

\section{Variational-hemivariational inequalities. An equilibrium approach 6.1 A class of variational-hemivariational inequalities}

Let $V$ be a reflexive Banach space endowed with norm $\|\cdot\|$. Further, suppose we have a bounded domain $D \subset \mathbb{R}^{d}$ (with $d=1,2,3$ ) and a linear compact operator $\chi: V \rightarrow L^{p}(D)$ $(1<p<\infty)$. Important particular cases of this framework are:

(1) $V=W_{0}^{1, p}(\Omega)$ with Lipschitz domain $D=\Omega \subset \mathbb{R}^{d}$, where $d=2,3$, and $\chi$ is the canonical injection that is compact by the Rellich-Kondrachev theorem; see [1], [52, Theorem 1.7.4];

(2) $V=W_{\Gamma_{D}, 0}^{1, p}(\Omega):=\left\{v \in W^{1, p}(\Omega): v \mid \Gamma_{D}=0\right\}$ with Lipschitz domain $\Omega \subset \mathbb{R}^{d}$ with $d=2,3$, where $\Gamma_{D}$ and $D=\Gamma_{C}$ are boundary parts of $\partial \Omega$, and $\chi$ is the trace map that is compact by the trace theorem; see [38, Theorem 6.10.5], [56].

In this setting, we treat a class of variational-hemivariational inequalities with the following main ingredients:

- a convex closed subset $C$ of $V$ that can explicitly describe constraints

- an extended real-valued convex l.s.c. function $f: C \rightarrow(-\infty,+\infty], f \not \equiv \infty$, that can implicitly describe constraints

- a monotone nonlinearity given by a monotone operator $A: V \rightarrow V^{*}$

- a nonmonotone nonlinearity given by the generalized Clarke directional derivative of a locally Lipschitz function $j$ (the so-called "superpotential") that gives rise to a p.m. bifunction

To make the latter ingredient precise, let us consider a function $j: D \times \mathbb{R}^{d} \rightarrow \mathbb{R}$ such that $j(\cdot, \xi): D \rightarrow \mathbb{R}$ is measurable on $D$ for all $\xi \in \mathbb{R}^{d}$ and $j(s, \cdot): \mathbb{R}^{d} \rightarrow \mathbb{R}$ is locally Lipschitz on $\mathbb{R}^{d}$ for almost all (a.a.) $s \in D$. Let $j^{0}(s, \cdot ; \cdot)$ stand for the generalized Clarke directional derivative [18] of $j(s, \cdot)$, and let $\partial j(s, \xi):=\partial j(s, \cdot)(\xi)$ denote the generalized subdifferential of $j(s, \cdot)$ at the point $\xi$ in the sense of Clarke [18]. Then we define the bifunction $\varphi$ on $V \times V$ by

$$
\varphi(u, v)=\int_{D} j^{0}(s,(\chi u)(s) ;(\chi v)(s)-(\chi u)(s)) d s, \quad \forall u, v \in V .
$$

Finally, let $l \in V^{*}$ be a continuous linear form, which - similarly as $C$ and $f$ - will be specified further.

With this data, we consider the nonlinear variational-hemivariational inequality (HVI): Find $u \in C$ such that

$$
\langle A(u), v-u\rangle+\varphi(u, v)+f(v) \geq f(u)+\langle l, v-u\rangle \quad \forall v \in C .
$$

From Theorem 5.3(B) we derive the following existence result, which extends [15, Theorem 3.1] from hemivariational inequalities with bilinear forms to nonlinear variationalhemivariational inequalities. 
Theorem 6.1 Let an operator $A: V \rightarrow V^{*}$ be hemicontinuous and strongly monotone, that is,

$$
\langle A(v)-A(w), v-w\rangle \geq c_{A}\|v-w\|^{p} \quad \forall v, w \in V,
$$

where $c_{A}>0$ and $1<p<\infty$. Suppose that there exist positive constants $c_{1}$ and $c_{2}$ such that for a.a. $s \in D$, all $\xi \in \mathbb{R}^{d}$, and all $\eta \in j j(s, \xi)$, the following inequalities hold:

(i) $|\eta| \leq c_{1}(1+|\xi|)$;

(ii) $\eta^{T} \xi \geq-c_{2}|\xi|$.

Then there exists a solution to the HVI (9).

Proof

First, note that conditions (i) and (ii) ensure that the integral in (8) is well defined. Indeed, it follows from (i) and (ii) that for a.a. $s \in D$,

$$
\left|j^{0}(s, \xi ; \varsigma)\right|=\left|\max _{\eta \in \partial j(s, \xi)} \eta^{T} \varsigma\right| \leq \max _{\eta \in \partial j(s, \xi)}|\eta||\varsigma| \leq c_{1}(1+|\xi|)|\varsigma| \quad \forall \xi, \varsigma \in \mathbb{R}^{d}
$$

and

$$
j^{0}(s, \xi ;-\xi)=\max _{\eta \in \partial j(s, \xi)} \eta^{T}(-\xi) \leq c_{2}|\xi| \quad \forall \xi \in \mathbb{R}^{d}
$$

Moreover, thanks to [49, Lemma 1] and [31, Lemma 4.1], the functional $\varphi$ is p.m. and satisfies

$$
\varphi(u, 0) \leq c_{3} \operatorname{meas}^{1 / 2}(D)\|\chi\|\|u\| \quad \forall u \in V
$$

for some positive constant $c_{3}$. Hence the given bifunction $\varphi$ satisfies the linear growth condition (6). Finally, relation (5) is satisfied with $G(t)=c_{A} t^{p-1}$. Thus Theorem 5.3(B) applies to arrive at the conclusion.

An example is the following scalar variational problem that models nonmonotone contact problems and employs the $p$-Laplace operator.

Example 6.1 Let $\Omega \subset \mathbb{R}^{d}$ be a bounded domain with Lipschitz boundary $\partial \Omega(d=2,3)$. Decompose $\partial \Omega$ into three disjoint parts $\Gamma_{D}, \Gamma_{N}$, and $\Gamma_{C}, \partial \Omega=\bar{\Gamma}_{D} \cup \bar{\Gamma}_{N} \cup \bar{\Gamma}_{c}$, where zero boundary conditions are imposed on the Dirichlet part $\Gamma_{D}$ with positive measure, surface tractions $\mathbf{t} \in L^{2}\left(\Gamma_{N} ; \mathbb{R}^{d}\right)$ act on the Neumann part $\Gamma_{N}$, and on the contact part $\Gamma_{C}$ (with positive measure), a nonmonotone, generally multivalued boundary condition holds. We consider the reflexive Banach space $V=W_{\Gamma_{D}, 0}^{1, p}(\Omega)=\left\{v \in W^{1, p}(\Omega): v \mid \Gamma_{D}=0\right\}$ with $p \geq 2$, endowed with the norm

$$
\|v\|_{1, p}=\left\{\int_{\Omega}\|\nabla v\|^{p} d x\right\}^{1 / p}
$$

Let $A: V \rightarrow V^{*}$ be the operator defined for $u, v \in V$ by

$$
\langle A(u), v\rangle=\int_{\Omega}\|\nabla u\|^{p-2} \nabla u \cdot \nabla v d x .
$$


Hence for some positive constant $c_{A}$, we have

$$
\langle A(u)-A(v), v-u\rangle \geq c_{A}\|u-v\|_{1, p}^{p}
$$

for all $u, v \in V$. It follows that the operator $A: V \rightarrow V^{*}$ is monotone and relation (5) is satisfied with $G(t)=c_{A} t^{p-1}$.

We prescribe nonmonotone, generally set-valued boundary conditions on $\Gamma_{c}$ via the bifunction $\varphi: V \times V \rightarrow \mathbb{R}$ given by

$$
\varphi(u, v):=\int_{\Gamma_{c}} j^{0}(\gamma u(s) ; \gamma v(s)-\gamma u(s)) d s \quad \forall u, v \in V .
$$

Here $j^{0}(\xi ; \eta)$ is the generalized Clarke derivative [18] of a locally Lipschitz function $j$ : $\mathbb{R}^{d} \rightarrow \mathbb{R}$ at $\xi \in \mathbb{R}^{d}$ in the direction $\eta \in \mathbb{R}^{d}$. Further, $\gamma$ stands for the linear compact trace operator from $V \subset W^{1, p}(\Omega)$ into $L^{p}\left(\Gamma_{c}\right)$ with the norm $\|\gamma\|$.

Moreover, the given right-hand side $f_{0} \in L^{2}(\Omega), f_{1} \in L^{p}\left(\Gamma_{N}\right)$ defines the linear form

$$
\langle l, v\rangle:=\int_{\Omega} f_{0} \cdot v d x+\int_{\Gamma_{N}} f_{1} \cdot \gamma v d s
$$

Let $C$ be a nonempty closed convex subset of $V$ to be specified further.

With this data, we consider the nonlinear variational-hemivariational inequality (HVI): Find $u \in C$ such that

$$
\langle A(u), v-u\rangle+\varphi(u, v) \geq\langle l, v-u\rangle \quad \forall v \in C .
$$

We need the following growth conditions on the locally Lipschitz superpotential $j: \mathbb{R} \rightarrow$ $\mathbb{R}$ :

(i) $|\eta| \leq c_{I}(1+|\xi|)$ for all $\eta \in \partial j(\xi)$ with $c_{I}>0$;

(ii) $\eta(-\xi) \leq c_{\text {II }}|\xi|$ for all $\eta \in \partial j(\xi)$ with $c_{\text {II }}>0$.

According to [48, Lemma 1], the bifunction $\varphi: V \times V \rightarrow \mathbb{R}$ is well-defined, pseudomonotone, and upper-semicontinuous, and relation (6) is satisfied with

$$
c_{1}:=c_{\mathrm{II}} \operatorname{meas}\left(\Gamma_{c}\right)^{1 / 2}\|\gamma\|
$$

Thus altogether this example fits to the frame described above, and Theorem 5.3(B) applies to conclude the existence of a solution to the HVI (15).

Finally, in this subsection, we comment on uniqueness of the solution to the HVI (15).

Remark 6.1 Introduce the upper Lipschitz condition on the bifunction $\varphi$ : There exists a constant $c_{\varphi}>0$ such that

$$
\varphi(u, v)+\varphi(v, u) \leq c_{\varphi}\|u-v\|^{2} \quad \forall u, v \in V
$$


Then as it is easily seen, the smallness condition $c_{\varphi}<c_{A}$ guarantees the uniqueness; see, e.g., [46, Theorem 5.1] and [47, Sect. 5.1, Theorem 1]. However, this crucial smallness condition is very abstract. For a detailed study of this condition for a class of locally Lipschitz functions $j$ that takes the jumps of $\partial j$ into account, we refer to [47, Sect. 5.1].

\subsection{A more general class of variational-hemivariational inequalities}

In this subsection, we turn to a more general class of variational-hemivariational inequalities on a reflexive Banach space $V$. Following [57, Sect. 5.4], there are the following ingredients: a convex closed subset $C \subset V$, an operator $A: C \rightarrow V^{*}$, a bifunction $\psi: C \times C \rightarrow \mathbb{R}$, and a locally Lipschitz function $J: C \rightarrow \mathbb{R}$ with the Clarke generalized directional derivative $J^{0}$. In addition, we introduce an extended real-valued convex and 1.s.c. function $f: C \rightarrow(-\infty,+\infty], f \not \equiv \infty$, and consider the following extended real-valued HVI problem: Find an element $\hat{u} \in C$ such that

$$
\langle A(\hat{u}), v-\hat{u}\rangle+\psi(\hat{u}, v)-\psi(\hat{u}, \hat{u})+J^{0}(\hat{u} ; v-\hat{u})+f(v) \geq f(\hat{u}) \quad \forall v \in C .
$$

We require the following conditions on the data $A, \psi, J$ :

$\left[C_{A}\right] A: C \rightarrow V^{*}$ is such that

(1) $A$ is hemicontinuous;

(2) there exists $m_{A}>0$ such that

$$
\left\langle A\left(v_{1}\right)-A\left(v_{2}\right), v_{1}-v_{2}\right\rangle \geq m_{A}\left\|v_{1}-v_{2}\right\|^{2} \quad \text { for all } v_{1}, v_{2} \in C \text {. }
$$

$\left[C_{\psi}\right] \psi: C \times C \rightarrow \mathbb{R}$ is such that

(1) $\psi(u, \cdot): C \rightarrow \mathbb{R}$ is convex and l.s.c. on $C$ for all $u \in C$;

(2) there exists $\alpha_{\psi}>0$ such that there holds the Lipschitz condition

$$
\begin{aligned}
& \psi\left(u_{1}, v_{2}\right)-\psi\left(u_{1}, v_{1}\right)+\psi\left(u_{2}, v_{1}\right)-\psi\left(u_{2}, v_{2}\right) \\
& \quad \leq \alpha_{\psi}\left\|u_{1}-u_{2}\right\|\left\|v_{1}-v_{2}\right\| \text { for all } u_{1}, u_{2}, v_{1}, v_{2} \in C .
\end{aligned}
$$

$\left[C_{J}\right] J: C \rightarrow \mathbb{R}$ is such that

(1) $J$ is locally Lipschitz;

(2) there exists $\alpha_{J}>0$ such that

$$
J^{0}\left(v_{1} ; v_{2}-v_{1}\right)+J^{0}\left(v_{2} ; v_{1}-v_{2}\right) \leq \alpha_{J}\left\|v_{1}-v_{2}\right\|^{2}
$$

Theorem 6.2 Assume that conditions $\left[C_{A}\right],\left[C_{\psi}\right]$, and $\left[C_{J}\right]$ hold. Moreover, suppose the smallness condition

$$
\alpha_{\psi}+\alpha_{J}<m_{A}
$$

Then the HVI (18) is uniquely solvable.

Proof Introduce

$$
\varphi_{1}(u, v):=\psi(u, v)-\psi(u, u) ; \quad \varphi_{2}(u, v):=J^{0}(u ; v-u),
$$




$$
\Phi(u, v):=\langle A(u), v-u\rangle+\varphi_{1}(u, v)+\varphi_{2}(u, v) \quad \forall u, v \in C .
$$

Then for all $u \in C, \Phi(u, \cdot)$ is convex and l.s.c. on $C$, and $\Phi(u, u)=0$.

The bifunction $\varphi_{2}$ is hemicontinuous, that is, for all $u, v \in C$, the function $t \in[0,1] \rightarrow$ $\left.\varphi_{2}(\operatorname{tv}+(1-t) u, v)=J^{0}(\operatorname{tv}+(1-t) u ;(1-t)(v-u))\right)$ is u.s.c. (upper semicontinuous) at $t=0$, since $(x . w) \rightarrow J^{0}(x ; w)$ is u.s.c.; see [18, Prop.2.1.1(b)].

Also, the bifunction $\varphi_{1}$ is hemicontinuous. To see this, use the shorthand $w_{t}:=\operatorname{tv}+(1-$ $t) u$ for fixed $u, v \in C$. Then $w_{t} \rightarrow u$ as $t \rightarrow 0$, and

$$
\limsup _{t \rightarrow 0}\left[\psi(u, u)-\psi\left(u, w_{t}\right)\right] \leq 0
$$

since $\psi(u, \cdot)$ is l.s.c. Then write

$$
\begin{aligned}
\varphi_{1}\left(w_{t}, v\right)-\varphi_{1}(u, v)= & \psi\left(w_{t}, v\right)-\psi\left(w_{t}, w_{t}\right)-\psi(u, v)+\psi(u, u) \\
= & {\left[\psi(u, u)-\psi\left(u, w_{t}\right)\right] } \\
& +\left\{\psi\left(u, w_{t}\right)+\psi\left(w_{t}, v\right)-\psi\left(w_{t}, w_{t}\right)-\psi(u, v)\right\} .
\end{aligned}
$$

Thanks to the Lipschitz condition, the term in the curled brackets is bounded above by

$$
\alpha_{\psi}\left\|u-w_{t}\right\|\left\|v-w_{t}\right\|=\alpha_{\psi} t(1-t)\|v-u\|^{2} \rightarrow 0 \quad \text { as } t \rightarrow 0 .
$$

In virtue of (20), the hemicontinuity follows. Thus the bifunction $\Phi$ is also hemicontinuous. Next, we estimate using $\left[C_{A}\right](2),\left[C_{\psi}\right](2)$, and $\left[C_{J}\right](2)$ :

$$
\begin{aligned}
\Phi(u, v)+\Phi(v, u)= & \langle\mathrm{Au}-\mathrm{Av}, v-u\rangle+\psi(u, v)-\psi(u, u)+\psi(v, u)-\psi(v, v) \\
& +J^{0}(u ; v-u)+J^{0}(v ; u-v) \\
\leq & -\left(m_{A}-\alpha_{\psi}-\alpha_{J}\right)\|u-v\|^{2} \quad \forall u, v \in C .
\end{aligned}
$$

Hence the smallness condition (19) implies that the bifunction $\Phi$ is strongly monotone. Further, (21) and (19) imply with the lower bound (7) for the convex l.s.c. function $f$, shown in the proof of Theorem 5.3 , that $f(\cdot)-\Phi\left(\cdot, u_{0}\right)$ for some $u_{0} \in C$ satisfies the asymptotic coercivity condition (4). Thus Theorem 5.2 yields the existence of a solution to the HVI (18).

To show the uniqueness, we employ the standard argument: Let $\bar{u}_{1}, \bar{u}_{2} \in C$ be two different solutions to the HVI (18). Then insert $v=\bar{u}_{2}$, respectively, $v=\bar{u}_{1}$ into the HVI (18). Add the resulting inequalities

$$
\begin{aligned}
& \Phi\left(\bar{u}_{1}, \bar{u}_{2}\right)+f\left(\bar{u}_{2}\right) \geq f\left(\bar{u}_{1}\right), \\
& \Phi\left(\bar{u}_{2}, \bar{u}_{1}\right)+f\left(\bar{u}_{1}\right) \geq f\left(\bar{u}_{2}\right)
\end{aligned}
$$

to obtain

$$
\Phi\left(\bar{u}_{1}, \bar{u}_{2}\right)+\Phi\left(\bar{u}_{2}, \bar{u}_{1}\right) \geq 0
$$

which contradicts (21) and (19). 
Some remarks on the variational problems in this section and on the previous existence result are in order.

\section{Remark 6.2}

1) When we drop $\varphi$ in (9) and when we drop $\psi$ and $J^{0}$ in (18), we obtain a standard elliptic variational inequality with nonlinear monotone operator $A$. On the other hand, dropping the operator $A$ in (9) and in (18) leads in view of the $\varphi$ and $J^{0}$ terms, respectively, to a hemivariational inequality. Therefore, following [57], (9) and (18) are called elliptic variational-hemivariational inequalities. In contrast, further in (18), there is a convex l.s.c. function $\psi(, \cdot)$ on $C$ depending on $v \in C$. So problem (18) can also be considered as a quasi-hemivariational inequality (of the second kind), and thus Theorem 6.2 extends [12, Theorem 4.16].

2) In comparison with [57, Theorem 84, Sect. 5.4], it suffices in Theorem 6.2 all the maps to be defined on $C$ only and to demand the single smallness condition (19). The proof of Theorem 6.2 by the equilibrium approach is shorter than that of [12, Theorem 4.16] and that of [57, Theorem 84, Sect. 5.4], which rely on existence results for classical variational inequalities and need an extra fixed point argument.

3) It is worth noting that because of the restrictive feature of the smallness condition (19), in the application to static contact problems in [57, Chap. 8] when (see [57, (4.9), p.126]), analogously to (14),

$$
J(v)=\int_{\Gamma_{c}} j(s, \gamma v(s)) d \Gamma,
$$

the norm of the trace operator $\gamma: V \rightarrow L^{2}\left(\Gamma_{c} ; \mathbb{R}^{d}\right)$ enters the smallness condition; see [57, Lemma 8, p.126], but the compactness of $\gamma$ is not required. In contrast, Theorem 6.1 and Example 6.1 demand the compactness of $\gamma$.

\section{A frictional unilateral contact problem}

In this section, we apply the main result of Sect. 5 to study the existence of solutions for a broad class of variational-hemivariational inequalities that model unilateral contact problems with nonmonotone, generally set-valued friction laws in solid mechanics. Similar nonmonotone friction phenomena occur with adhesion/cohesion and delamination problems in material science; see, for instance, $[20,35,45]$ and the references therein.

Let us consider a deformable body which occupies the Lipschitz domain $\Omega \subset \mathbb{R}^{d}(d=$ $2,3)$. The boundary $\Gamma=\partial \Omega$ is decomposed into three disjoint open parts such that $\Gamma=$ $\overline{\Gamma_{D}} \cup \overline{\Gamma_{N}} \cup \overline{\Gamma_{C}}$ with meas $\left(\Gamma_{D}\right)>0$ and meas $\left(\Gamma_{C}\right)>0$. Suppose that the process is static and, in addition, the body is subjected to volume forces of density $f_{0}$ in $\Omega$ and to surface tractions of density $f_{1}$ on $\Gamma_{N}$. On $\Gamma_{C}$ the body is in frictional unilateral contact with a rigid obstacle (foundation). We model the friction by a boundary condition in the tangential direction involving Clarke's generalized gradient and leading to the study of a nonlinear variational-hemivariational inequality problem.

Let $\mathbb{S}^{d}$ be the space of second-order symmetric tensors on $\mathbb{R}^{d}$. For $\mathbf{u}=\left(u_{i}\right), \mathbf{v}=\left(v_{i}\right) \in \mathbb{R}^{d}$, and $\sigma, \tau \in \mathbb{S}^{d}$, the inner product and the Euclidean norm on $\mathbb{S}^{d}$ and $\mathbb{R}^{d}$ are, respectively, $\mathbf{u} \cdot \mathbf{v}=u_{i} v_{i},\|\mathbf{u}\|=(\mathbf{u} \cdot \mathbf{u})^{1 / 2}, \sigma \cdot \tau=\sigma_{\mathrm{ij}} \tau_{\mathrm{ij}},\|\sigma\|=(\sigma \cdot \sigma)^{1 / 2}$. We also use the usual notation for the normal components and the tangential parts of vectors and tensors, respectively, given by $u_{N}=\mathbf{u} \cdot \mathbf{n}, \mathbf{u}_{T}=\mathbf{u}-u_{N} \mathbf{n}, \sigma_{N}=\sigma_{\mathrm{ij}} n_{i} n_{j}$, and $\sigma_{T}=\sigma \mathbf{n}-\sigma_{N} \mathbf{n}, \partial_{N} \mathbf{u}=\nabla \mathbf{u} \cdot \mathbf{n}$, where 
$\mathbf{n}=\left(n_{i}\right)$ represents the outward unit normal vector to the Lipschitz boundary $\partial \Omega$, which is defined almost everywhere.

We consider the following contact problem for a nonlinear elastic body with unilateral constraints and nonmonotone friction condition, where the friction coefficient is slip dependent.

Problem $\left(\mathcal{P}_{1}\right)$ : Find a displacement field $\mathbf{u}=\left(u_{i}\right): \Omega \rightarrow \mathbb{R}^{d}$ and a stress field $\sigma=\left(\sigma_{\mathrm{ij}}\right):$ $\Omega \rightarrow \mathbb{S}^{d}$ such that

$$
\begin{aligned}
& \sigma(\mathbf{u})=\mathcal{A}(\varepsilon(\mathbf{u})) \quad \text { in } \Omega, \\
& \operatorname{Div} \sigma(\mathbf{u})+\mathbf{f}_{0}=\mathbf{0} \quad \text { in } \Omega, \\
& \mathbf{u}=\mathbf{0} \quad \text { on } \Gamma_{D}, \\
& \sigma(\mathbf{u}) \mathbf{n}=\mathbf{f}_{N} \quad \text { on } \Gamma_{N}, \\
& u_{N} \leq g, \quad \sigma_{N}(\mathbf{u}) \leq 0, \quad \sigma_{N}(\mathbf{u})\left(u_{N}-g\right)=0 \quad \text { on } \Gamma_{C}, \\
& -\sigma_{T}(\mathbf{u}) \in \mu\left(\left\|\mathbf{u}_{T}\right\|\right) \partial j\left(\mathbf{u}_{T}\right) \quad \text { on } \Gamma_{C} .
\end{aligned}
$$

Equation (22) represents the constitutive law for nonlinear elastic materials. The contact is assumed to be static, and hence we use the equilibrium equation (23), $\mathbf{f}_{0}$ being the body force density. Equations (24) and (25) are the classical displacement and traction boundary conditions: the body is fixed on $\Gamma_{D}$, and surface tractions of density $\mathbf{f}_{N}$ are applied on $\Gamma_{N}$. Assuming that the foundation is perfectly rigid, we have the unilateral Signorini condition (26) on the surface $\Gamma_{C}$, where $g$ describes the gap between body and foundation. Relation (27) exhibits the friction law, where $\partial j$ denotes the generalized gradient of the given locally Lipschitz function $j$, and $\mu$ is the friction coefficient, which is assumed to be a positive function on $\Gamma_{C}$. The function $\mu$ may depend on the slip, that is, on the tangential displacement. For details on mathematical description of static contact models, see, for instance, $[41,56]$.

We require the following conditions on the nonlinear elastic operator $\mathcal{A}$, the superpotential $j$, and the friction coefficient $\mu$.

$\left[C_{\mathcal{A}}\right] \mathcal{A}: \Omega \times \mathbb{S}^{d} \rightarrow \mathbb{S}^{d}$ satisfies

(1) $\mathcal{A}(\cdot, \varepsilon)$ is measurable on $\Omega$ for all $\varepsilon \in \mathbb{S}^{d}$;

(2) $\mathcal{A}(x, \cdot)$ is hemicontinuous for a.a. $x \in \Omega$;

(3) There exist $\alpha_{0} \in L^{2}(\Omega), \alpha_{0} \geq 0$, and a constant $\alpha_{1}>0$ such that

$$
\|\mathcal{A}(x, \varepsilon)\| \leq \alpha_{0}(x)+\alpha_{1}\|\varepsilon\| \quad \text { for all } \varepsilon \in \mathbb{S}^{d} \text { and a.a. } x \in \Omega \text {; }
$$

(4) There exists a constant $\alpha_{2} \geq 0$ such that for all $\varepsilon_{1}, \varepsilon_{2} \in \mathbb{S}^{d}$ and a.a. $x \in \Omega$,

$$
\left(\mathcal{A}\left(x, \varepsilon_{1}\right)-\mathcal{A}\left(x, \varepsilon_{2}\right)\right) \cdot\left(\varepsilon_{1}-\varepsilon_{2}\right) \geq \alpha_{2}\left\|\varepsilon_{1}-\varepsilon_{2}\right\|^{2} .
$$

$\left[C_{j}\right] j: \Gamma_{C} \times \mathbb{R}^{d} \rightarrow \mathbb{R}$ is such that

(1) $j(x, \cdot)$ is locally Lipschitz on $\mathbb{R}^{d}$ for a.a. $x \in \Gamma_{C}$;

(2) $j(\cdot, \xi)$ is measurable on $\Gamma_{C}$ for all $\xi \in \mathbb{R}^{d}$;

(3) $|\eta| \leq a(1+|\xi|)$ for all $\eta \in \partial j(x, \xi), \xi \in \mathbb{R}^{d}$ and a.a. $x \in \Gamma_{C}$ with $a>0$;

(4) $\eta \cdot(-\xi) \leq b|\xi|$ for all $\eta \in \partial j(x, \xi), \xi \in \mathbb{R}^{d}$ and a.a. $x \in \Gamma_{C}$ with $b>0$. 
$\left[C_{\mu}\right] \mu: \Gamma_{C} \times \mathbb{R}^{+} \rightarrow \mathbb{R}^{+}$is such that

(1) $\mu(\cdot, s)$ is measurable on $\Gamma_{C}$ for all $s \in \mathbb{R}^{+}$;

(2) There exists $L_{\mu}>0$ such that

$$
\left|\mu\left(x, s_{1}\right)-\mu\left(x, s_{2}\right)\right| \leq L_{\mu}\left|s_{1}-s_{2}\right|, \quad \text { for all } s_{1}, s_{2} \in \mathbb{R}^{+} \text {and a.a. } x \in \Gamma_{C}
$$

(3) There exists $\mu_{0}>0$ such that $\mu(x, s) \leq \mu_{0}$ for all $s \in \mathbb{R}^{+}$and a.a. $x \in \Gamma_{C}$.

Moreover, we suppose that the following regularity conditions are satisfied by the gap function and the densities of the body forces and surface traction:

$$
g \in L^{2}\left(\Gamma_{c}\right), \quad \mathbf{f}_{0} \in L^{2}\left(\Omega ; \mathbb{R}^{d}\right), \quad \mathbf{f}_{N} \in L^{2}\left(\Gamma_{N} ; \mathbb{R}^{d}\right) .
$$

To give the weak formulation of problem $\left(\mathcal{P}_{1}\right)$, we consider the following Hilbert spaces:

$$
V=\left\{\mathbf{v} \in H^{1}\left(\Omega ; \mathbb{R}^{d}\right): \mathbf{v}=\mathbf{0} \text { on } \Gamma_{D}\right\}, \quad H=L^{2}\left(\Omega ; \mathbb{R}^{d}\right), \quad \mathcal{H}=L^{2}\left(\Omega ; \mathbb{S}^{d}\right) .
$$

The inner products over the spaces $\mathcal{H}$ and $V$ are given respectively by

$$
\langle\sigma, \tau\rangle_{\mathcal{H}}=\int_{\Omega} \sigma_{\mathrm{ij}}(x) \tau_{\mathrm{ij}}(x) d x \quad \text { for } \sigma, \tau \in \mathcal{H} \text {, and }\langle\mathbf{u}, \mathbf{v}\rangle_{V}=\langle\varepsilon(\mathbf{u}), \varepsilon(\mathbf{v})\rangle_{\mathcal{H}} \text { for } \mathbf{u}, \mathbf{v} \in V .
$$

Let $\gamma: V \rightarrow L^{2}\left(\Gamma ; \mathbb{R}^{d}\right)$ be the trace operator, which is continuous and compact, and

$$
\|\gamma(\mathbf{v})\|_{L^{2}\left(\Gamma ; \mathbb{R}^{d}\right)} \leq\|\gamma\|\|\mathbf{v}\|_{V} \quad \text { for all } \mathbf{v} \in V
$$

where $\|\gamma\|$ denotes the norm of $\gamma$. We define the load functional $\mathbf{l} \in V^{*}$ by

$$
\langle\mathbf{l}, \mathbf{v}\rangle=\left\langle\mathbf{f}_{0}, \mathbf{v}\right\rangle_{H}+\left\langle\mathbf{f}_{N}, \gamma(\mathbf{v})\right\rangle_{L^{2}\left(\Gamma_{N} ; \mathbb{R}^{d}\right)} \text { for } \mathbf{v} \in V .
$$

In the following, we omit the symbol $\gamma$ and simply write $\mathbf{v}$ for the trace of an element $\mathbf{v} \in V$ when considered on a boundary part. The set of admissible displacement fields is given by

$$
C=\left\{\mathbf{v} \in V: v_{N} \leq g \text { on } \Gamma_{C}\right\}
$$

which is a closed and convex subset of $V$.

Let $A: V \rightarrow V$ be the nonlinear operator defined by $\langle A(\mathbf{u}), \mathbf{v}\rangle_{V}:=\langle\mathcal{A}(\varepsilon(\mathbf{u})), \varepsilon(\mathbf{v})\rangle_{\mathcal{H}}$ for $\mathbf{u}, \mathbf{v} \in V$. Then the weak formulation of problem $\left(\mathcal{P}_{1}\right)$ is the following.

Problem $\left(\mathcal{P}_{2}\right)$ : Find a displacement field $\overline{\mathbf{u}} \in C$ such that

$$
\begin{aligned}
& \langle A(\overline{\mathbf{u}}), \mathbf{v}-\overline{\mathbf{u}}\rangle_{V}+\int_{\Gamma_{C}} \mu\left(x,\left\|\overline{\mathbf{u}}_{T}(x)\right\|\right) j^{0}\left(x, \overline{\mathbf{u}}_{T}(x) ; \mathbf{v}_{T}(x)-\overline{\mathbf{u}}_{T}(x)\right) d \Gamma(x) \\
& \quad \geq\langle\mathbf{l}, \mathbf{v}-\overline{\mathbf{u}}\rangle \quad \forall \mathbf{v} \in C .
\end{aligned}
$$

Conditions $\left[C_{j}\right]$ and $\left[C_{\mu}\right]$ ensure that the integral in (29) is well defined.

By conditions $\left[C_{\mathcal{A}}\right]$, the operator $A: V \rightarrow V^{*}$ is well-defined, hemicontinuous and uniformly monotone. Introduce the bifunction $\varphi: V \times V \rightarrow \mathbb{R}$ by

$$
\varphi(\mathbf{u}, \mathbf{v})=\int_{\Gamma_{C}} \mu\left(x,\left\|\overline{\mathbf{u}}_{T}(x)\right\|\right) j^{0}\left(x, \overline{\mathbf{u}}_{T}(x) ; \mathbf{v}_{T}(x)-\overline{\mathbf{u}}_{T}(x)\right) d \Gamma(x) \quad \forall \mathbf{u}, \mathbf{v} \in V .
$$


Then by a similar reasoning as in the proof of [31, Lemma 4.1], we can show that conditions $\left[C_{j}\right]$ and $\left[C_{\mu}\right]$ imply that $\varphi$ is p.m. In addition, $\varphi$ satisfies condition (6). Thus, proceeding similarly as above in Example 6.1 and as in the proof of Theorem 6.1, we derive from Theorem 5.3(B) the existence of a solution $\overline{\mathbf{u}} \in C$ to problem $\left(\mathcal{P}_{2}\right)$.

\section{Concluding remarks. An outlook}

There is a vast body of literature on the KKM principle with a lot of extensions and ramifications. When these results generalize and as well rely on the classical KKM principle, then they are obviously equivalent in a broad sense. Nevertheless, further results that, similarly to Theorem 2.2, unify some ramifications and reveal the close relationship among them; see, for example, [53].

In our equilibrium approach to variational inequalities with set-valued operators, we focused on variational inequalities of the first kind. Extended real-valued set-valued variational inequalities of the second kind with a lower semicontinuous convex function as a further ingredient are also worth studying.

Further, we focused on a class of variational-hemivariational inequalities with application to static contact problems. Whereas these problems are of interest in their own right, they are also an important building block in the study of more complicated timedependent problems encountered with contact problems in viscoelasticy and viscoplasticity; here we refer, for example, to [19, 33, 54, 56, 57].

We also focused on coercive equilibrium problems and coercive variational inequalities. With loss of coerciveness, the existence can be guaranteed under extra conditions for the right-hand side (see the recent paper [32] and the references therein), and the BrowderTikhonov regularization methods come into play; see, for example, [3, 13, 16, 24, 39].

Acknowledgements

The author thanks the three anonymous reviewers for their careful reading of the text.

Funding

Not applicable

Abbreviations

KKM, Knaster-Kuratowski-Mazurkiewicz; pm, pseudomonotone; HVI, variational-hemivariational inequality; I.s.c., lower semicontinuous; u.s.c., upper semicontinuous; VI, variational inequality; a.a., almost all. All these abbreviations are explained in the text.

Availability of data and materials

Not applicable

\section{Declarations}

Competing interests

The author declares that he has no competing interests.

Authors' contributions

The author read and approved the final manuscript.

\section{Publisher's Note}

Springer Nature remains neutral with regard to jurisdictional claims in published maps and institutional affiliations.

Received: 22 September 2021 Accepted: 6 January 2022 Published online: 31 January 2022

References

1. Adams, R.A., Fournier, J.F.F.: Sobolev Spaces, 2nd edn. Elsevier/Academic Press, Amsterdam (2003) 
2. Agarwal, R.P., Meehan, M., O'Regan, D.: Fixed Point Theory and Applications. Cambridge University Press, Cambridge (2009)

3. Alleche, B., Radulescu, V.D., Sebaoui, M.: The Tikhonov regularization for equilibrium problems and applications to quasi-hemivariational inequalities. Optim. Lett. 9, 483-503 (2015)

4. Ansari, Q.H., Lin, Y.C., Yao, J.C.: General KKM theorem with applications to minimax and variational inequalities. J. Optim. Theory Appl. 104, 41-57 (2000)

5. Aubin, J.P.: Mathematical Methods of Game and Economic Theory. North-Holland, Amsterdam $(1979,1982)$

6. Berge, C.: Espaces Topologiques, Fonctions Multivoques, 2nd edn. Dunod, Paris (1966)

7. Blum, E., Oettli, W.: From optimization and variational inequalities to equilibrium problems. Math. Stud. $63,123-145$ (1994)

8. Brézis, H.: Équations et inéquations non linéaires dans les espaces vectoriels en dualité. Ann. Inst. Fourier 18, 115-175 (1968)

9. Brézis, H., Nirenberg, L., Stampacchia, G.: A remark on Ky Fan's minimax principle. Boll. Unione Mat. Ital. 6, 293-300 (1972)

10. Browder, F.E.: On the unification of the calculus of variations and the theory of monotone nonlinear operators in Banach spaces. Proc. Natl. Acad. Sci. USA 56, 419-425 (1966)

11. Browder, F.E.: Nonlinear operators and nonlinear equations of evolution in Banach spaces. Nonlinear functional analysis. In: Proc. Sympos. Pure Math., Vol. XVIII, Part 2, Chicago, III, 1968, pp. 1-308 (1976)

12. Capatina, A.: Variational Inequalities and Frictional Contact Problems. Advances in Mechanics and Mathematics, vol. 31. Springer, Cham (2014)

13. Chadli, O.: A Tikhonov-type regularization for Brezis pseudomonotone equilibrium problems in Banach spaces. J. Appl. Numer. Optim. 3(2), 285-295 (2021)

14. Chadli, O., Ansari, Q.H., Yao, J.C.: Mixed equilibrium problems and anti-periodic solutions for nonlinear evolution equations. J. Optim. Theory Appl. 168(2), 410-440 (2016)

15. Chadli, O., Chbani, Z., Riahi, H.: Some existence results for coercive and noncoercive hemivariational inequalities. Appl. Anal. 69(1-2), 125-131 (1998)

16. Chadli, O., Schaible, S., Yao, J.C.: Regularized equilibrium problems with application to noncoercive hemivariational inequalities. J. Optim. Theory Appl. 121(3), 571-596 (2004)

17. Chowdury, M.S.R., Tan, K.-K.: Generalization of Ky Fan's minimax inequality with applications to generalized variational inequalities for pseudo-monotone operators and fixed point theorems. J. Math. Anal. Appl. 204, 910-929 (1996)

18. Clarke, F.: Optimization and Nonsmooth Analysis. Wiley, New York (1983)

19. Cocou, M.: A class of dynamic contact problems with Coulomb friction in viscoelasticity. Nonlinear Anal., Real World Appl. 22, 508-519 (2015)

20. Dao, M.N., Gwinner, J., Noll, D., Ovcharova, N.: Nonconvex bundle method with application to a delamination problem. Comput. Optim. Appl. 65(1), 173-203 (2016)

21. Ding, X.P., Tarafdar, E.: Generalized variational-like inequalities with pseudomonotone set-valued mappings. Arch. Math. (Basel) 74, 302-313 (2000)

22. Fan, K.: A generalization of Tychonoff's fixed-point theorem. Math. Ann. 142, 305-310 (1961)

23. Fan, K.: A minimax inequality and applications, inequalities, III. In: Proc. Third Sympos., Univ. California, Los Angeles, Calif, 1969, pp. 103-113 (1972)

24. Giannessi, F., Khan, A.A.: Regularization of non-coercive quasi variational inequalities. Control Cybern. 29(1), 91-110 (2000)

25. Goeleven, D., Motreanu, D., Dumont, Y., Rochdi, M.: Variational and Hemivariational Inequalities: Theory, Methods and Applications, Vol. II: Unilateral Problems. Kluwer, Dordrecht (2003)

26. Gwinner, J: Nichtlineare Variationsungleichungen mit Anwendungen. PhD Thesis, Universität Mannheim (1978)

27. Gwinner, J: On the existence and approximation of solutions of pseudomonotone variational inequalities. In: Henn R., Korte, B., Oettli, W. (eds.) Optimization and Operations Research. Lect. Notes Econ. Math. Syst., vol. 157, pp. 117-125. Springer, Berlin (1978)

28. Gwinner, J.: On fixed points and variational inequalities - a circular tour. Nonlinear Anal. 5, 565-583 (1981)

29. Gwinner, J.: An extension lemma and homogeneous programming. J. Optim. Theory Appl. 47, 321-336 (1985)

30. Gwinner, J.: A note on pseudomonotone functions, regularization, and relaxed coerciveness. Nonlinear Anal. 30 4217-4227 (1997)

31. Gwinner, J., Ovcharova, N.: From solvability and approximation of variational inequalities to solution of nondifferentiable optimization problems in contact mechanics. Optimization 64(8), 1683-1702 (2015)

32. Gwinner, J., Ovcharova, N.: A Gårding inequality based unified approach to various classes of semi-coercive variational inequalities applied to non-monotone contact problems with a nested max-min superpotential. Minimax Theory Appl. 5(1), 103-128 (2020)

33. Han, W., Sofonea, M.: Quasistatic Contact Problems in Viscoelasticity and Viscoplasticity. AMS/IP Studies in Advanced Mathematics, vol. 30. American Mathematical Society/International Press, Providence (2002)

34. Hartman, P., Stampacchia, G.: On some non-linear elliptic differential-functional equations. Acta Math. 115, 271-310 (1966)

35. Hintermüller, M., Kovtunenko, V.A., Kunisch, K.: Obstacle problems with cohesion: a hemivariational inequality approach and its efficient numerical solution. SIAM J. Optim. 21, 491-516 (2011)

36. Kalmoun, E.M.: On Ky Fan's minimax inequalities, mixed equilibrium problems and hemivariational inequalities. JPAM. J. Inequal. Pure Appl. Math. 2, Article ID 12 (2001)

37. Kneser, H.: Sur un théorème fondamental de la théorie des jeux. C. R. Acad. Sci. Paris 234, 2418-2420 (1952)

38. Kufner, A., John, O., Fučik, S.: Function Spaces. Academia, Prague (1977)

39. Liu, Z:: Browder-Tikhonov regularization of non-coercive evolution hemivariational inequalities. Inverse Probl. 21, 13-20 (2005)

40. Maugeri, A., Raciti, F.: On existence theorems for monotone and nonmonotone variational inequalities. J. Convex Anal. 16, 899-911 (2009)

41. Migórski, S., Ochal, A., Sofonea, M.: Nonlinear Inclusions and Hemivariational Inequalities: Models and Analysis of Contact Problems. Springer, New York (2013) 
42. Minty, G.J.: On the generalization of a direct method of the calculus of variations. Bull. Am. Math. Soc. 73, 315-321 (1967)

43. Moré, J.J.: Coercivity conditions in nonlinear complementarity problems. SIAM Rev. 16, 1-16 (1974)

44. Naniewicz, Z., Panagiotopoulos, P.D.: Mathematical Theory of Hemivariational Inequalities and Applications. Dekker, New York (1995)

45. Nesemann, L., Stephan, E.P.: Numerical solution of an adhesion problem with FEM and BEM. Appl. Numer. Math. 62, 606-619 (2012)

46. Ovcharova, N.: On the coupling of regularization techniques and the boundary element method for a hemivariational inequality modelling a delamination problem. Math. Methods Appl. Sci. 40, 60-77 (2017)

47. Ovcharova, N., Banz, L.: Coupling regularization and adaptive hp-BEM for the solution of a delamination problem. Numer. Math. 137, 303-337 (2017)

48. Ovcharova, N., Gwinner, J.: A study of regularization techniques of nondifferentiable optimization in view of application to hemivariational inequalities. J. Optim. Theory Appl. 162(3), 754-778 (2014)

49. Ovcharova, N., Gwinner, J.: On the discretization of pseudomonotone variational inequalities with an application to the numerical solution of the nonmonotone delamination problem. In: Rassias, T.M., et al. (eds.) Optimization in Science and Engineering, pp. 393-405. Springer, New York (2014)

50. Panagiotopoulos, P.D.: Hemivariational Inequalities. Applications in Mechanics and Engineering. Springer, Berlin (1993)

51. Panagiotopoulos, P.D.: Inequality Problems in Mechanics and Application - Convex and Nonconvex Energy Functions. Birkhäuser, Basel (1998)

52. Papageorgiou, N.S., Radulescu, V.D., Repovs, D.: Nonlinear Analysis - Theory and Methods. Springer Monographs in Mathematics. Springer, Cham (2019)

53. Park, S.: Evolution of the 1984 KKM theorem of Ky Fan. Fixed Point Theory Appl. 2012, 146 (2012)

54. Shillor, M., Sofonea, M., Telega, J.J.: Models and Analysis of Quasistatic Contact. Lecture Notes in Physics, vol. 655 Springer, Berlin (2004)

55. Simons, S.: Minimax and variational inequalities. Are they of fixed-point or Hahn-Banach type? Game theory and mathematical economics. In: Proc. Semin., Bonn/Hagen, 1980, pp. 379-388 (1981)

56. Sofonea, M., Matei, A.: Mathematical Models in Contact Mechanics. London Mathematical Society Lecture Note Series, vol. 398. Cambridge University Press, Cambridge (2012)

57. Sofonea, M., Migórski, S.: Variational-Hemivariational Inequalities with Applications. Monographs and Research Notes in Mathematics. CRC Press, Boca Raton (2018)

58. Stampacchia, G.: Variational inequalities, theory and applications of monotone operators. In: Proc. NATO Advanced Study Inst., Venice, Oderisi-Gubbio, pp. 101-192 (1969)

59. Zeidler, E.: Nonlinear Functional Analysis and Its Applications. II/B Nonlinear Monotone Operators. Springer, New York (1990) Translated from the German by the author, Boron, Leo F.

\section{Submit your manuscript to a SpringerOpen ${ }^{\circ}$ journal and benefit from:}

- Convenient online submission

- Rigorous peer review

- Open access: articles freely available online

- High visibility within the field

- Retaining the copyright to your article

Submit your next manuscript at $\boldsymbol{s p r i n g e r o p e n . c o m ~}$ 